\title{
How toxic are gold nanoparticles? The State-of-the-Art
}

\author{
Ilaria Fratoddi ${ }^{1}(\varangle)$, lole Venditti ${ }^{1}$, Cesare Cametti ${ }^{2}$, and Maria Vittoria Russo ${ }^{1}$ \\ ${ }^{1}$ Dipartimento di Chimica, Universita' di Roma "La Sapienza", Piazzale A. Moro 5, I-00185 Rome, Italy \\ ${ }^{2}$ Dipartimento di Fisica, Universita' di Roma "La Sapienza", Piazzale A. Moro 5, I-00185 Rome, Italy and INFM CNR-SOFT, Unita' di Roma1
}

\author{
Received: 1 October 2014 \\ Revised: 11 December 2014 \\ Accepted: 13 December 2014 \\ (c) Tsinghua University Press \\ and Springer-Verlag Berlin \\ Heidelberg 2015

\section{KEYWORDS} \\ gold nanoparticles, \\ nanospheres, \\ nanorods, \\ nanocages, \\ nanostars, \\ toxicity
}

\begin{abstract}
With the growing interest in the applications of gold nanoparticles in biotechnology and their physiological effects, possible toxicity of gold nanoparticles is becoming an increasingly important issue. A large number of studies carried out over the past few years under a variety of experimental conditions and following different protocols have produced conflicting results, leading to divergent views about the actual safety of gold nanoparticles in human applications.

This work is intended to provide an overview of the most recent experimental results and thereby summarize current state-of-the-art. Rather than presenting a comprehensive review of the available literature in this field, which would be impractically broad, we have selected representative examples of both in vivo and in vitro studies, which clearly demonstrate the need for urgent and rigorous standardization of experimental protocols. Despite their significant potential, the safety of gold nanoparticles is highly controversial at this time, and important concerns have been raised that need to be properly addressed. Factors such as shape, size, surface charge, coating, and surface functionalization are expected to influence the interactions of particles with biological systems to a different extent, resulting in different outcomes and influencing the potential of gold nanoparticles for biomedical applications.

Moreover, despite continuous attempts to establish a correlation between structure of the particles and their interactions with biological systems, we are still far from elucidating the toxicological profile of gold nanoparticles in an indisputable manner. This review is intended to contribute towards this goal, offering a number of suggestions on how to achieve the systematization of data on the most relevant physico-chemical parameters, which govern and control the toxicity of gold nanoparticles at cellular and whole-organism levels.
\end{abstract}

\section{Introduction}

Gold in its bulk form has long been considered an inert, non-toxic, biocompatible, and noble metal with a number of therapeutic (and even medicinal) properties. However, when the size of the particle decreases to

Address correspondence to ilaria.fratoddi@uniroma1.it 
the nanometer scale, gold behaves very differently compared to its bulk form. Its safety, as a promising material for biomedical applications, becomes questionable in nanoparticle form, with important concerns raised in the assessment of risks for humans. The $a$ priori assumption that gold nanoparticles (AuNPs) are intrinsically biocompatible must be rejected.

On the other hand, the term "toxicity" is itself rather vague. From a theoretical point of view, toxicology is related to the adverse effects that a generic substance exerts on living organisms. In this context, it must be recognized that, with exposure to sufficient quantities, all materials can be toxic. This basic principle of toxicology was expressed by Paracelsus more than five centuries ago, in his statement that many drugs that are beneficial at low doses are toxic at high doses [1]. The identification of the relevant dosimetry is therefore a very important aspect of the evaluation of particle toxicity $[2,3]$.

The basic question to be addressed is: How toxic are AuNPs at the concentrations at which they may potentially be used for therapeutic purposes? At present, even with a considerable number of published reports, this question remains basically unresolved.

AuNPs have been widely used in medical and biological research, with applications that include targeted delivery of drugs [4], optical bioimaging of cells and tissues [5], imaging and diagnosis of a number of diseases [6], as an intravenous contrast agent for imaging and noninvasive detection of lung cancer, and many other uses [7]. With widespread applications, many labs have tried to investigate the safety of AuNPs from different perspectives.

Among these investigations, a large body of experimental work has confirmed the non-toxicity of AuNPs [8-10]. Conversely, just as much contradicting evidence was presented by other research groups, revealing AuNPs to be toxic [11-13].

An illustrative example of the confused and complicated situation in the research field is the study performed by Villiers et al. [14], who extracted bone marrow-generated dendritic cells from C57BL/6 mice and analyzed their viability after incubation in the presence of AuNPs with a mean size of $10 \mathrm{~nm}$ and a $\zeta$ potential of $-13.0 \mathrm{mV}$ at $\mathrm{pH}=7.4$. The results of this study showed these AuNPs to be non-cytotoxic, even at high concentrations. However, further analysis at the intracellular level revealed notable amounts of AuNPs accumulating in endocytic compartments. The secretion of cytokines was found to be significantly modified after such internalization, indicating a potential perturbation in the immune response. These findings show that these AuNPs were not completely bio-inert (and biocompatible), even if they exhibited no apparent toxicity.

Beyond the wide variability of experimental conditions and the substantial discrepancies found in a considerable part of the published results, the general opinion is that naked AuNPs (i.e., as synthesized) are significantly toxic both in vitro and in vivo, while appropriate coating may attenuate their harmful effects [15].

This intriguing scenario is even more confusing if one considers the different parameters that may influence the potential toxic effects of AuNPs, and the observed toxicity that is directly related to five distinct factors: i) surface chemistry, ii) coating materials, iii) size, iv) shape, and v) biological target evaluated [16].

For each of these physico-biochemical parameters, AuNPs offer extraordinarily wide range of possibilities. For example, AuNP size may vary from few nanometers to hundreds of nanometers. AuNPs exist in a variety of different shapes, including nanospheres, nanorods, nanocages, nanoshells, and nanostars, among others. Finally, the relatively straightforward synthesis allows the surface of AuNPs to be functionalized in a number of different ways by using a variety of coating agents, including small molecules such as citrate, surfactants such as cetyltrimethylammonium bromide (CTAB), or polymers and proteins. Recently, polyvinylpyrrolidone (PVP) has been added to this class of molecules, suggesting the possibility of producing safer nanomaterials [17].

The kind of effects that nanoparticles may induce must also be considered. Even if we confine ourselves to cellular toxicity, there are two specific forms of cell death, which have increasingly received attention in relation to the exposure of cells to AuNPs [18]. The first of these processes is the controlled cell death (apoptosis). The second is a pathological process 
of cell death (necrosis) that occurs in response to externally induced toxicity.

Due to the differences in experimental methods employed, the extraordinary variety of sizes and functionalities of AuNPs, and the variability of cell lines, there is a lack of consensus on nanoparticle toxicity at this time. Moreover, a persistent fundamental question remains, since it is still not clear whether the occurrence toxicity arises in association with the chemical functionalization of nanoparticles or is simply a result of the small particle size, which favors cell internalization. There is lack of clear correlation between toxicity and either of the two parameters, resulting in a current lack of clear understanding of the intrinsic effects of nanoparticles.

Consequently, standardization of experimental approaches, such as the choice of model (cell lines, animal species), exposure conditions (cell confluence, exposure duration, nanoparticle-concentration ranges, and dosing increments), and physico-chemical characteristics of AuNPs is necessary to allow comparisons to be made between investigations conducted by different researchers in a conclusive and comprehensive way.

At present, the major obstacle is the significant discrepancy in experimental conditions under which toxic effects and bio-distribution of AuNPs have been evaluated by individual non-correlated investigations. In most of these studies, only few specific parameters have been monitored, without a systematic control of others factors with a well-designed protocol.

In order to effectively compare different experimental results, it is highly desirable to standardize the protocols used, as far as particle size, shape, purity, intracellular stability, and surface charge and chemistry are concerned, as well as cell types, since different cell lines can react quite differently to the same type of nanoparticles.

An interesting progress in this direction comes from the work published by Pompa et al. [19], who proposed a systematic and reproducible evaluation of nanoparticle toxicology in living systems based on physical assessment and quantification of the toxic effects of AuNPs through an experimental determination of key parameters affecting the toxicity outcomes. These authors were able to define different regions in the multi-parametric space of toxicity. This approach may pave the way to a systematic classification of nanomaterials, leading to important developments in risk assessment that can be used in a wide range of nanomedical applications.

Numerous excellent reviews on AuNP toxicity have been published so far [15, 20-30], with one important recent review in particular dealing with the evaluation of toxic effects [31]. As pointed out by these authors [31], the interpretation of the results of AuNP safety assessments is complicated by the considerable variability in: "i) Types of AuNPs, ii) stabilizing coating agents, iii) physicochemical parameters of the NPs (diameter, surface charge, surface topography, surface area), iv) incubation conditions (time and concentration), v) type of cells used, vi) type of assay used or vii) possible interference of the NPs with the assay readout". We have reproduced here the exact phrasing used by these authors [31] because it captures in a striking way the core of the problem. However, their assertion makes it rather difficult to define the optimal method that can be used to study AuNP cytotoxicity.

In this review, we present the results of a series of recent experiments evaluating the in vivo and in vitro cytotoxicity of AuNPs, ranging in diameter from 1 (a cluster of few atoms) to $200 \mathrm{~nm}$, and with shapes that include nanospheres, nanorods, nanoshells, nanocages, and nanostars. Rather than providing an exhaustive coverage of the most recent studies, we chose to focus on the evidence demonstrating how differences in experimental conditions under which toxicity effects are evaluated reduce the possibility of reaching general conclusions regarding the effective safety of AuNPs. Based on this scenario, there is a pressing need for a common protocol and a predictive paradigm that can be used to screen multiple overlapping factors. In light of these considerations, we summarize the main physico-chemical parameters associated with AuNPs, which, based on the data published to date, influence their toxicity. We provide a series of suggestions with the aim of progressing towards a standardized protocol, which would yield a systematic and reproducible assessment of AuNP toxicity with precise control of different parameters, which govern this complex phenomenon. 


\section{Parameters affecting toxicology evalua- tion: General considerations}

Before entering the core of the problem, we will examine, with a critical point of view, some of the parameters, which are currently inadequately defined, contributing to the conflicting results.

The toxicity of nanoparticles is commonly expressed as the particle concentration causing $50 \%$ of growth inhibition in a cell culture $\left(\mathrm{IC}_{50}\right)$. As far as this parameter is concerned, equimolar doses should be presented, rather than numerical particle concentrations, in comparing the toxicity of various AuNPs, since the tendency of nanoparticles to aggregate renders the particle number concentration practically meaningless. Close attention should also be paid to the interactions between nanoparticles and biological fluids, which may favor particle aggregation $[32,33]$.

Commonly used media include cell culture medium (with or without serum), phosphate-buffered saline (PBS), $0.9 \%$ sodium chloride, plasma, and whole blood. While cells are typically exposed to nanoparticles in media containing no serum or a reduced amount of serum, this is sometimes not possible since the cells require minimal serum levels to maintain normal viability. Presence of serum greatly favors particle aggregation, making particle concentration even more irrelevant.

In vitro cytotoxicity is studied using various animal cell cultures, with the fibroblasts of human skin (HeLa), human leukemia (K562), human hepatocarcinoma (HepG2), human breast carcinoma (SK-BR-3), and other cell lines commonly used. Immortalized cell lines like HeLa cells are commonly used to compare the cytotoxicity of nanoparticles varying in size and surface chemistry [34, 35]. Data on in vivo cytotoxicity were recently reviewed by Johnston et al. [30], who presented a detailed analysis of particle characteristics and a description of different mechanisms responsible for the observed toxicity.

Selection of the appropriate cytotoxicity assay is vital for the accurate assessment of nanoparticle toxicity. Various assays can be used to study the toxic effects of nanoparticles on cell cultures, including lactate dehydrogenase (LDH) leakage, 3-(4,5-dimethylthiazol-2-yl)-2,5-diphenyltetrazolium bromide (MTT) assay, and quantification of cytokine/chemokine production.

Following treatment with AuNPs, dead cells were imaged with the commonly used fluorescent dye propidium iodide (PI). Normally, fluorescent PI molecules cannot penetrate the cell membrane. However, in some of the experiments, PI molecules entered the cells during endocytosis of the nanoparticles, resulting in a false-positive result by overestimating the toxicity [36].

Surface charge is a key parameter of nanoparticles. Cationic nanoparticles are able to bind negatively charged DNA. When DNA binds to highly positive nanoparticles, it wraps around the nanoparticle and bends. This bending generally causes damage to DNA. Usually, hydrophobic ligands bind to the minor grooves and charged ligands can bind to both minor and major grooves, where high electrostatic and van der Waals interactions are required [37]. Positively charged nanoparticles with sufficiently high surface charge densities may attach to DNA irreversibly [38].

Anionic nanoparticles can be internalized within a cell through endocytotic pathways [39]. One important effect of particle surface functionalization is the change in the particle charge, since electrostatic interactions influence cellular uptake more strongly than hydrophobic or van der Waals interactions.

Variability in the data between different assays was found to result from the interference by factors such as nanoparticle-dye interactions and absorption of the dye by nanoparticles. Different experimental results have indicated that toxicity is highly dependent on the physico-chemical properties of nanoparticles. Moreover, the occurrence of false-positive and false-negative results highlights the importance of crosschecking the data using alternative assays to ensure the reliability of obtained results.

Specifically, particle size is an important parameter that affects the agglomeration, sedimentation, and diffusion of nanoparticles and in turn, affects the transport of nanoparticles into cells during the toxicity assay. On the other hand, researchers have also reported gold nanospheres (AuNSs) to be less cytotoxic than gold nanorods (AuNRs). These findings emphasize the importance of correlating specific sizes and shapes with toxic biological responses.

An often-overlooked aspect of nanoparticle toxicity 
profile is the clearance of nanoparticles from the body after their therapeutic effect is completed. As an example, a number of studies suggested that nanoparticles might be retained in the liver and spleen of mice for a long period of time, or even permanently. For example, Huang et al. [40] and Haimfeld et al. [41] observed the persistence of particles larger than $10 \mathrm{~nm}$ in liver and spleen of mice for up to 6 months with no apparent consequences. It is currently unknown if AuNPs completely clear from the body and what undesirable consequences their retention may provoke over longer time periods. A number of recent studies evaluating these aspects of human toxicology were reviewed by Gerber et al. [42], who asserted that the data available for predicting the hazard potential of AuNPs in humans is very limited at this time.

In the following review, we will summarize the main results concerning AuNP cytotoxicity outcomes from selected recent studies. The discussed effects are presented taking into account the nanoparticle shape.

\section{Gold nanospheres}

The synthesis, characterization, and functionalization of differently shaped AuNPs have been extensively reviewed by Dreaden et al. [43] and by us [44]. A number of facets of these techniques have been presented and discussed over the past years in a series of papers from our group [45-56].

An important effect observed when the effect of surface charge on toxicity and cellular uptake is considered is that nanoparticles have a positive effective surface charge upon preparation but are no longer cationic in the cellular media. Numerous plasma proteins spontaneously adsorb on nanoparticle surfaces, resulting in surface chemistry of the nanoparticles in growth media/plasma being quite different from that of the originally synthesized materials. Instead, nanoparticles adopt the physico-chemical properties of the adsorbed protein shell, called a protein corona [57-59]. A schematic representation of this relevant phenomenon is presented in Fig. 1. Although a complete understanding of nanoparticleprotein interaction is currently lacking [60], the

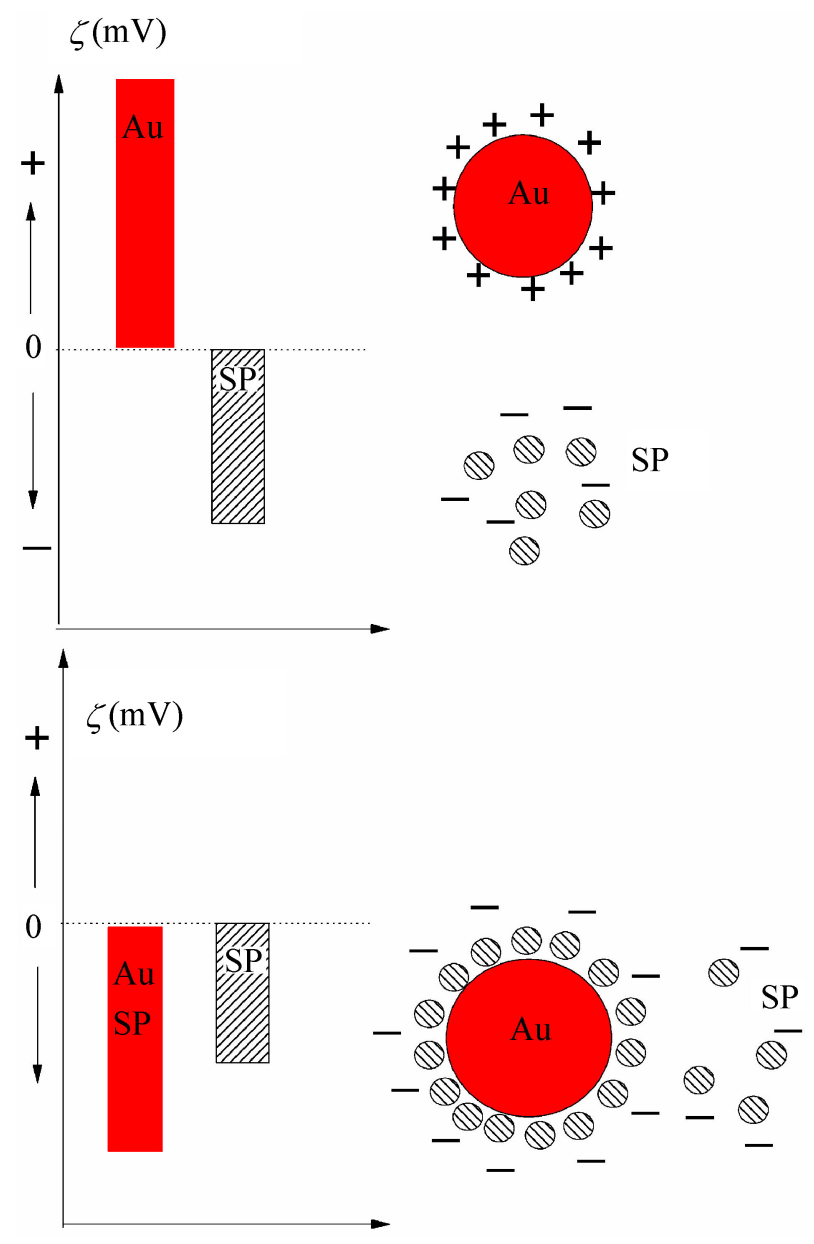

Figure 1 Schematic representation of the formation of protein corona on AuNP surface. Adsorption of serum proteins (SPs) onto the surface of AuNPs flips their effective surface charge from positive value (upper panel) to negative value (bottom panel). On the left, the change in the $\zeta$ potential is shown.

adsorbed protein layer is known to strongly influence cellular uptake and particle biodistribution, ultimately conditioning particle toxicity. The relevance of the protein corona for the biological impact of nanoparticles in vivo and in vitro has been discussed by Monopoli et al. [61] in the case of hydrophobic (sulfonated polystyrene, $\mathrm{PSOSO}_{3}$ ) and hydrophilic particles (silica, $\mathrm{SiO}_{2}$ ). General findings and conclusions of this study can be easily extended to AuNPs.

The effect of size of spherical AuNPs on their toxicity has been investigated in detail by various authors.

Water-soluble AuNPs stabilized by triphenylphosphine derivatives with sizes in the range from 0.8 to $15 \mathrm{~nm}$ were investigated by Pan et al. [34]. This group evaluated $\mathrm{IC}_{50}$ values from MTT assays and 
found that the cytotoxicity in different cell lines representing the principal barriers and lining cells of the body (connective tissue fibroblasts (L929), epithelial cells (HeLa), macrophages (J744A1), and melanoma cells (SK-Mel-28)), markedly depended on particle size. Particle size varied from 0.8 (cluster with 8 gold atoms) to $1.8 \mathrm{~nm}$ (cluster with 150 gold atoms). Cytotoxicity was investigated in both actively dividing cells (in the logarithmic growth phase) and quiescent cells (in the stationary phase). Figure 2 shows representative cytotoxicity observed during the logarithmic growth phase of the cell lines.

Particles $1.4 \mathrm{~nm}$ in size were found to be the most toxic, with $\mathrm{IC}_{50}$ values ranging from 30 to $56 \mu \mathrm{M}$, while particles of $0.8,1.2$, and $1.8 \mathrm{~nm}$ in size are less toxic at up to 60 - to 100 -fold higher concentrations. Moreover, $1.4 \mathrm{~nm}$ particles led to cell necrosis after $12 \mathrm{~h}$ of incubation. These results suggest a stringent and notable size dependency of the cytotoxicity, although the reason why a particular size produces more toxicity than the others remains unclear.

One of the best examples of the dependence of toxicity on AuNP concentration is provided by Pernodet et al. [62], who investigated the interaction

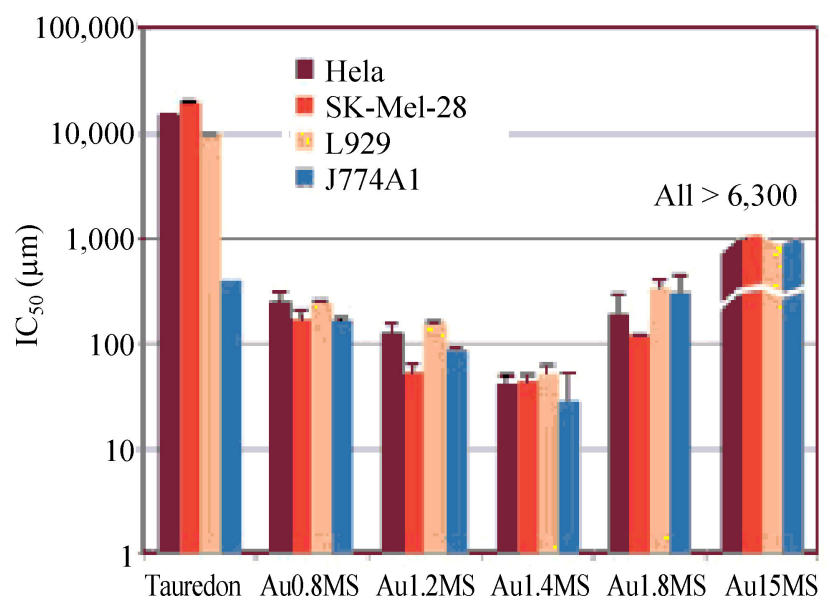

Figure 2 Cytotoxicity of AuNPs of different sizes (0.8, 1.2, 1.4, $1.8,15 \mathrm{~nm}$ ) during the logarithmic growth phase of 4 cell lines: HeLa cervical carcinoma epithelial cells (HeLa), melanoma cells (SK-Mel-28), mouse fibroblasts (L929), and mouse monocytic/ macrophage cells (J774A1). The $\mathrm{IC}_{50}$ values of AuNPs $1.4 \mathrm{~nm}$ in size were lowest across all cell lines, and AuNPs of smaller or larger size were observed to be progressively less cytotoxic. Reproduced with permission from Ref. [34], (C) 2007 WILEY-VCH Verlag GmbH \& Co. KGaA, Weinheim. between fibroblast cells and citrate-coated nanoparticles ( $1 \mathrm{~nm}$ in size) at concentrations ranging from 0.2 to $0.8 \mathrm{mg} \cdot \mathrm{mL}^{-1}$. Accumulation of different amounts of AuNP in vacuoles resulted in different degrees of damage to actin fibers, with density (at the top of the cell) ranging from $0.65 \mu \mathrm{m}^{-1}$ in controls to $0.1 \mu \mathrm{m}^{-1}$ at a particle concentration of $0.8 \mathrm{mg} \cdot \mathrm{mL}^{-1}$.

Since gold is one of the most electronegative metals, it is easily attracted to DNA grooves, which present a negative environment. Furthermore, AuNPs of approximately $1.4 \mathrm{~nm}$ diameter match almost perfectly the size of the major DNA groove, leading to strong potential for toxic effects of AuNPs, especially with smaller-sized particles [63].

Even if not directly connected to cytotoxicity, the intracellular uptake of spherical AuNPs depends critically on particle size.

While it is well-established that the small size of AuNPs plays a major role in the mechanism of their entry into cells, relatively little is known about their effects on human health. Chithrani et al. [64] incubated HeLa cells with citrate AuNPs of various sizes (diameters of 14, 30, 50, 74, and $100 \mathrm{~nm}$ ) for $6 \mathrm{~h}$ in Dulbecco minimum essential media (DMEM) plus $10 \%$ serum and evaluated the intracellular uptake using inductively coupled plasma atomic emission spectroscopy (ICP-AES). Maximum cellular uptake occurred at a nanoparticle size of $50 \mathrm{~nm}$, with uptake ranging from 500 to 6,000 particles per cell, depending on the degree of protein adsorption and the cell line used. In this case, non-specific adsorption of serum proteins onto the gold surface alters cellular uptake through receptor-mediated endocytosis.

Connor et al. [7] and later Murphy et al. [65] have examined the uptake and potential toxicity of a series of AuNPs in human leukemia cells. AuNSs varied in particle size $(4,12$, and $18 \mathrm{~nm}$ in diameter) and surface modifiers, including a range of anionic, neutral, and cationic groups: Citrate, cysteine, glucose, biotin, and CTAB. The K562 leukemia cell line was exposed to nanoparticles for 3 days, and the cell viability was measured using a colorimetric MTT assay. The results (Fig. 3) suggest that none of the spherical AuNPs were toxic to the human leukemia cells, with no detrimental effects on cell function observed at concentrations up to approximately $150 \mu \mathrm{M}$ in gold 


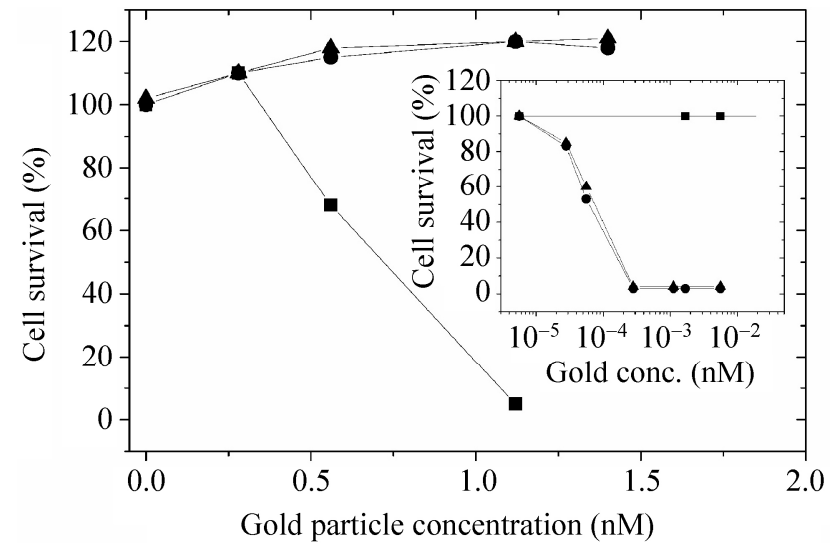

Figure 3 Percent survival of human K562 cells exposed to functionalized AuNPs (18 nm) for 3 days. Cells exposed to AuNPs containing citrate ( $\mathbf{(})$; Cells exposed to AuNPs containing biotin $(\bullet)$; cells exposed to $\mathrm{AuCl}_{4}$ precursor (•). Inset: cell exposed to AuNPs containing CTAB $(\mathbf{\bullet})$; cells exposed to CTAB alone $(\bullet)$ and cell exposed to AuNPs containing CTAB washed 3 times prior to incubation (•). Reproduced with permission from Ref. [7], (C) 2005 WILEY-VCH Verlag GmbH \& Co. KGaA, Weinheim.

atom concentration, despite the presence of cellular uptake of the particles, as confirmed by transmission electron microscopy (TEM) of cell slices.

The influence of size on in vivo tissue distribution of spherical-shaped AuNPs in rats has been recently investigated by De Jong et al. [66]. Rats were intravenously injected with AuNPs with diameters of 10, 50, 100, and $250 \mathrm{~nm}$ and AuNP concentration was quantitatively measured after $24 \mathrm{~h}$ using inductively coupled plasma mass spectrometry (ICP-MS) methods. Qualitatively similar results have been reported by Sonavane et al. [67] in tissues and organs of albino mice 24 hours after the administration of the dose. In Fig. 4, we present a synthesis of results of studies performed by De Jong et al. [66] and Sonavane et al. [67], which, taken together, confirm that the accumulation of AuNPs in various tissues is dependent on particle size. However, it is difficult to describe a well-defined behavior and the only conclusions that can be drawn is that relatively small particles (10-15 nm) show higher accumulation in all studied tissues, including blood, liver, lung, spleen, kidney, and heart. Relatively larger particles (200-250 nm) showed very low presence in organs such as blood, brain, and spleen.

Even if it is only indirectly related to toxicity, it is necessary to mention the work of Abdel Halim [68],
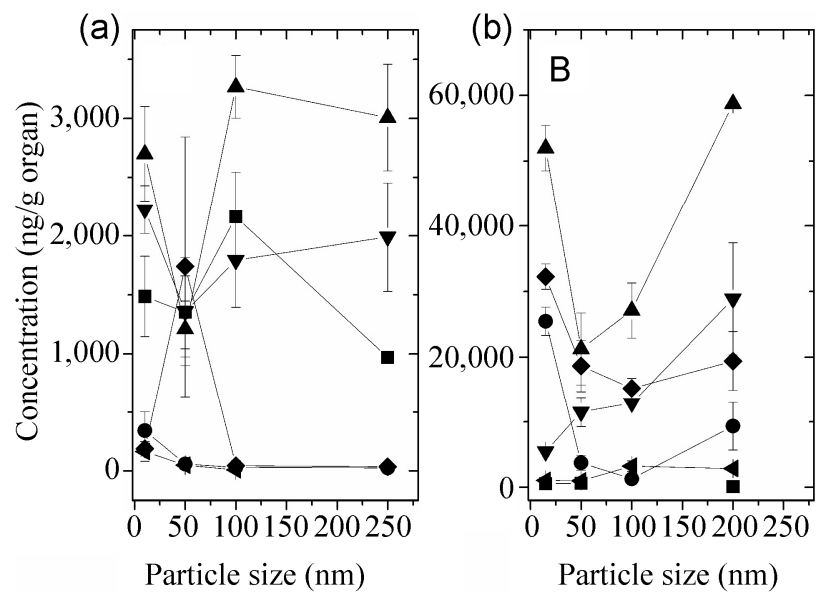

Figure 4 Concentration of AuNPs measured in different rat organs (expressed as ng/g organ): Blood (•); liver ( $\mathbf{\Delta})$; spleen ( $\boldsymbol{\nabla})$; lungs $(\diamond)$; kidney $(\bullet)$; heart $(\triangleleft)$. Left panel A: Reproduced with permission from Ref. [66], (c) 2008 Elsevier Ltd. Right panel B: Reproduced with permission from Ref. [67], (C) 2008 Elsevier B.V.

who investigated the in vivo accumulation of spherical (and spheroidal) AuNPs in multiple organs of rats. Accumulation of particles is considered to be an indication of nanoparticle toxicity. From the main results (summarized in Fig. 5), it is evident that greater toxicity is induced by AuNPs of smaller size, confirming the findings of De Jong et al. [66] and Sonavane et al. [67].

The effect of 15-nm citrate-capped AuNPs on the Drosophila melanogaster model has been investigated by Pompa et al. [69]. In this animal model, the investigators observed a strong reduction of life span and

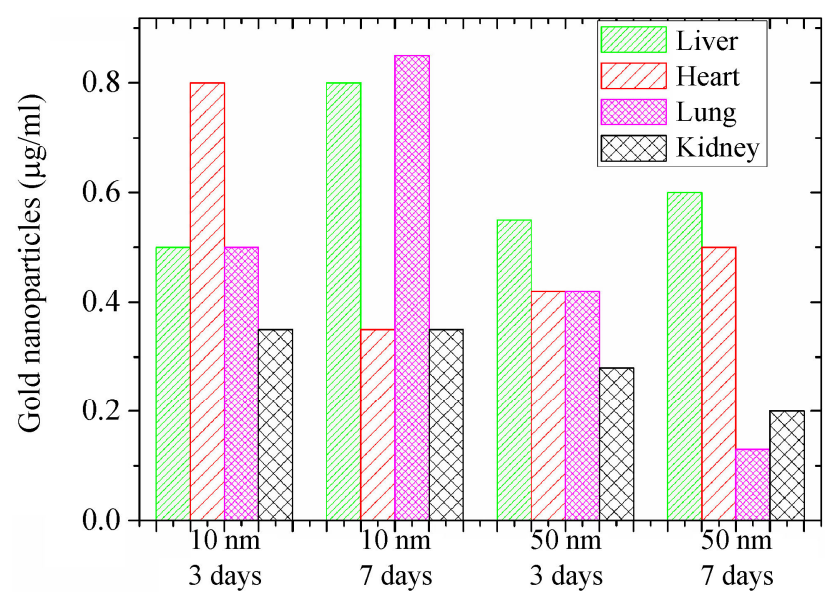

Figure 5 Concentration of AuNPs (10 and $50 \mathrm{~nm}$ in size) in different organs of rats after intraperitoneally administration of $50 \mu \mathrm{L}$ for 3 and 7 days. Reproduced with permission from Ref. [68], (c) 2012 Abdel Halim MAK. 
fertility, the presence of DNA fragmentation, and a significant over-expression of stress proteins following ingestion of $12 \mu \mathrm{g} / \mathrm{g}$ AuNPs per day. This example highlights how nanoparticles introduced into a complex systems, such as a living organism, are able to modify its functioning.

Application of polyethylene glycol (PEG) of nanoparticles (PEGylation) is commonly used to lower their cytotoxicity. However, PEG can lead to lower cellular internalization efficiency, thereby reducing the potential for using AuNPs as therapeutic agents.

Simpson et al. [70] have suggested that glutathione may be an attractive alternative to PEG in the design of AuNP therapeutics. Mice injected with glutathionecoated AuNPs did not exhibit any clinical signs of illness or morbidity (evaluated through histological analysis) at any concentration over the course of 6 weeks. Therefore, glutathione-coated AuNPs appear not to cause any toxic effects in passing through the kidneys, contrary to the findings of a previous study with tiopronin monolayer protected clusters (TMPC) evaluating the same concentrations [71].

AuNP stabilization with chitosan has been previously reported [72, 73]. Recently, Stefan et al. [74] presented a study evaluating the effects of AuNPs (12 and $22 \mathrm{~nm}$ in size) capped with chitosan on brain and liver tissue reactivity in male Wistar rats exposed to lipopolysaccharide (LPS) obtained from Escherichia coli through 8 daily intraperitoneal administrations. Their results demonstrated that chitosan-capped AuNPs of smaller size show protective effects against LPS-induced toxicity.

A detailed comparison of metabolites (Fig. 6) measured in the serum of animals treated with LPS with those of untreated control animals, showed that LPS induced toxicity with symptoms suggesting kidney dysfunction, as evidenced by the significant decrease in levels of urea nitrogen.

Conflicting results can arise from the variability in toxicity assays, cell lines, and physico-chemical properties of nanoparticles studied. For example, cytotoxicity results can vary with the cell line used. Citrate-capped AuNPs (13 nm in diameter) were found to be toxic in human carcinoma lung cell line, but not in the human liver carcinoma cell line at same dosage [9].

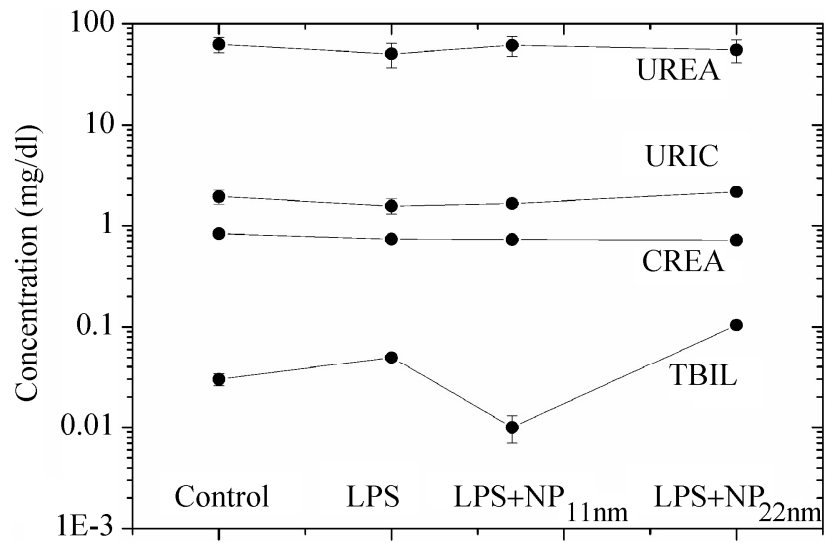

Figure 6 Biochemical parameters measured in the serum of rats treated with AuNPs (11 and $22 \mathrm{~nm}$ in size) after exposure to LPS. Reproduced with permission from Ref. [74], (C) 2012 Elsevier B.V.

Another point to be addressed is that in vitro and in vivo investigations are based on very different methodologies. In vitro, three-dimensional (3D) cell culture models have been used as a bridge between the in vitro two dimensional (2D) plated cell culture and the in vivo models [76]. In this context, Lee et al. [77] compared the toxicity of AuNPs in both 2D and 3D cell culture constructs. They used hydrogel inverted colloidal crystals as a cell growth substrate and human hepatocarcinoma cells to construct the 3D cell culture environment. They found that toxicity of both citrate- (anionic) and CTAB-capped (cationic) AuNPs was significantly reduced in the $3 \mathrm{D}$ environment, as compared with the 2D one [77]. These results suggest that in vitro studies alone are not adequate for the assessment of the toxicity of nanoparticles.

The effect of nanoparticles 5 and $15 \mathrm{~nm}$ in size at different concentrations $(10-300 \mu \mathrm{M})$ on Balb/3T3 mouse fibroblast cells in vitro has been investigated by Coradeghini et al. [75]. Cell cytotoxicity was evaluated by colony forming efficiency (CFE) assay [78] and trypan blue assay. The main findings, summarized in Fig. 7, were that toxicity occurs only following 72-h exposure to 5-nm AuNPs at concentrations higher than $50 \mu \mathrm{M}$ using the CFE assay, while no toxicity was observed, even at the highest concentration $(300 \mu \mathrm{M})$ and longest exposure time $(72 \mathrm{~h})$, when trypan blue assay is employed.

The above example is illustrative of two different concepts. First, it highlights that particle size plays an important role in AuNP toxicity. Although the 
CFE
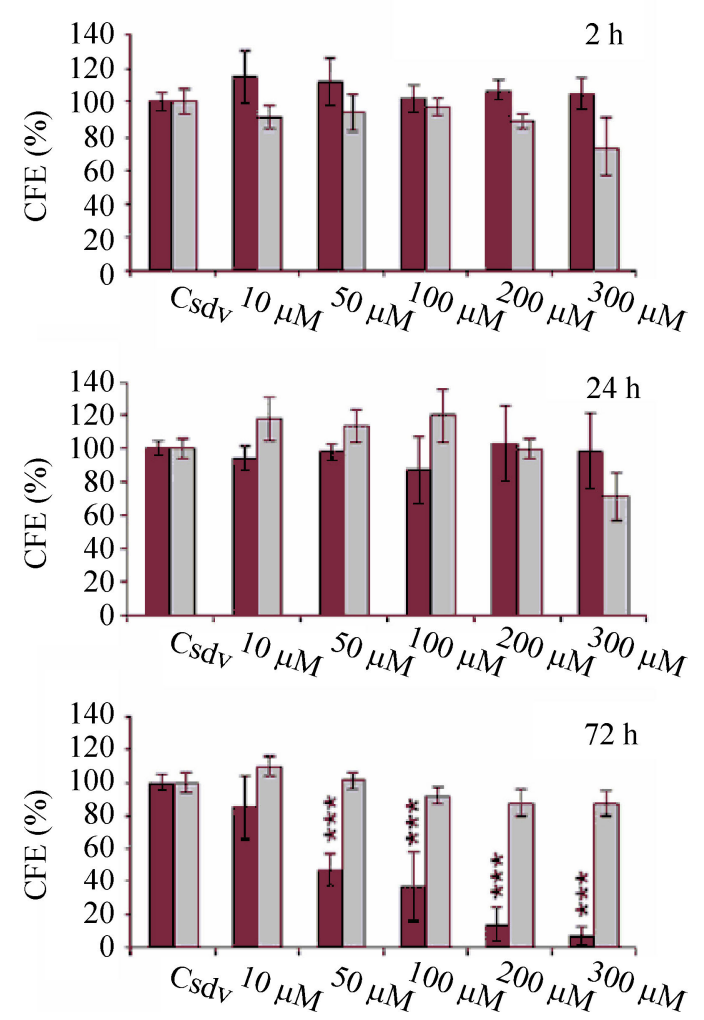

Trypan blue

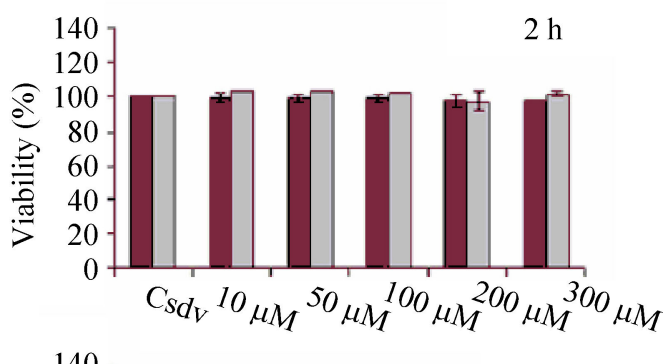

$24 \mathrm{~h}$
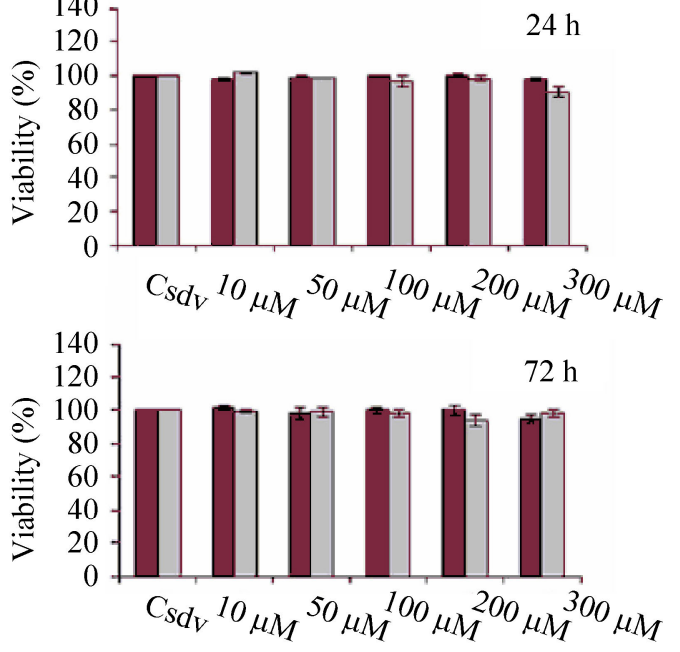

Figure 7 Toxicity of AuNPs with $5 \mathrm{~nm}$ (red histogram) and $15 \mathrm{~nm}$ (gray histogram) diameters in Balb/3T3 cells exposed for 2, 24, and $72 \mathrm{~h}$ to increasing concentration of AuNPs (10-300 $\mu \mathrm{M}$ ), evaluated using 2 different assays, colony forming efficiency (CFF) (on the left) and trypan blue exclusion test (on the right). AuNPs $5 \mathrm{~nm}$ induced cytotoxicity in Balb/3T3 cells following $72 \mathrm{~h}$ of exposure at concentrations higher than $50 \mu \mathrm{M}$. In the range of concentration and time-points tested, no cytotoxicity was observed in Balb/3T3 cells exposed to AuNPs $15 \mathrm{~nm}$. Reproduced with permission from Ref. [75], (c) 2012 Elsevier Ireland Ltd.

difference in sizes of AuNPs employed is very small, the overall biological response is significantly different. Second, different cytotoxicity results derived from the two different assays can be better understood when combined with the results obtained by different techniques. In this particular case, TEM analysis showed that AuNPs remain confined to vesicles without entering the nucleus, while inductively coupled plasma-mass spectroscopy (ICO-MS) revealed that the total gold content in cells increased in a timedependent manner. These additional findings can account, at least partially, for different cytotoxicity behaviors observed by these authors [75].

No toxicity of either non-functionalized or polyacrylamide-coated AuNPs $18 \mathrm{~nm}$ in size was reported by Salmaso et al. [87] in human breast adenocarcinoma cells. Likewise, no toxicity was detected by Qu et al. [88] with citrate-coated AuNPs,
10 and $50 \mathrm{~nm}$ in size, in embryonal fibroblasts up to a relatively high concentration of $300 \mu \mathrm{M}$.

These examples make it apparent that there is an urgent need for standardization of employed protocols to enhance our understanding of AuNPinduced cytotoxicity. A typical example illustrating this need is shown in Fig. 8, where different results concerning the viability of HeLa cells from the MTT assay from separate recent publications are collected together. The large number of parameters involved and the differences between studies in parameters studied makes it difficult to organize the available data in a completely intelligible way. We present the data ordered by particle size (which has been shown to be an important parameter determining both the efficiency of cellular internalization and cytotoxicity) and the concentration of AuNP. This choice implies that the characteristics of each AuNP formulation are 


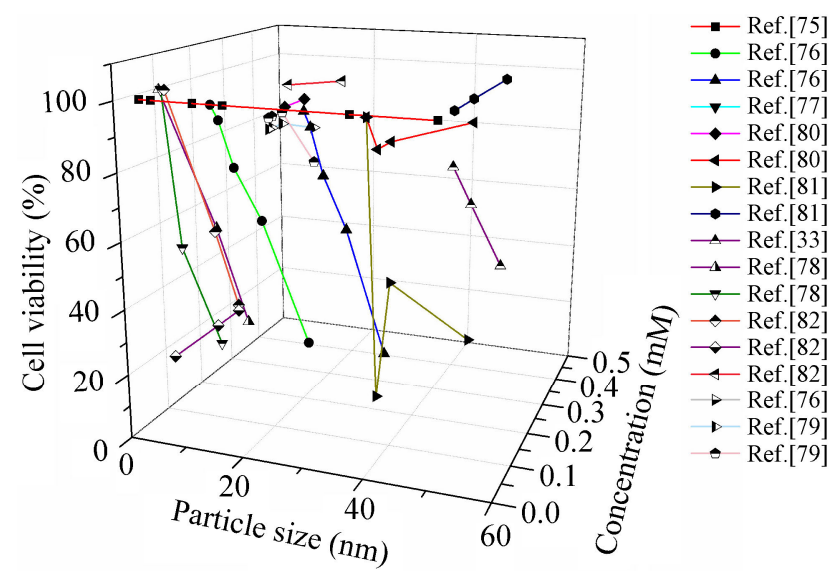

Figure 8 Viability data (derived from MTT assay) collected from recent literature on HeLa cells incubated with AuNPs of different sizes and at different concentrations. AuNPs are differently functionalized. Data taken from Refs. [34, 79-86].

determined by the differences in surface functionalization, ranging from naked nanoparticles to PEG-coated nanoparticles. As can be seen from the data, the large variability prevents the detection of any reasonable correlations among them, with the exception of a demonstrably higher toxic effect of larger particles, as compared to the smaller ones.

A further example is reported in Fig. 9, presenting data previously reported by Patra and Dasgupta [89] on the responses of cancer cells to a variety of classes of nanoparticles with different hydrodynamic diameters and $\zeta$ potentials, assessed in terms of percentage cell survival using the MTT assay. The left panel shows the dependence of the response on AuNP size, while the right panel shows the relationship with the $\zeta$ potential. It is evident from the presented data that the relative cell survival with AuNPs smaller than and larger than $50 \mathrm{~nm}$ in size is comparatively similar, despite the higher abundance of nanoparticles with small hydrodynamic diameters (Fig. 9, left panel, zones $\mathrm{A}$ and $\mathrm{C}$ ). However, the influence of the $\zeta$ potential on cell survival was observed to be slightly linear. The bottom left quadrant (left panel, zone B) has very few points compared to the bottom right quadrant, implying that, even with particle sizes larger than $50 \mathrm{~nm}$, nanoparticles can interact with cells if the $\zeta$ potential is in the permissible range.

This kind of analysis of different cytotoxic results highlights the importance of data comparison and aims to characterize (from a phenomenological point of view) the influence exerted by different parameters when interactions between nanoparticles and cells are taken into consideration.

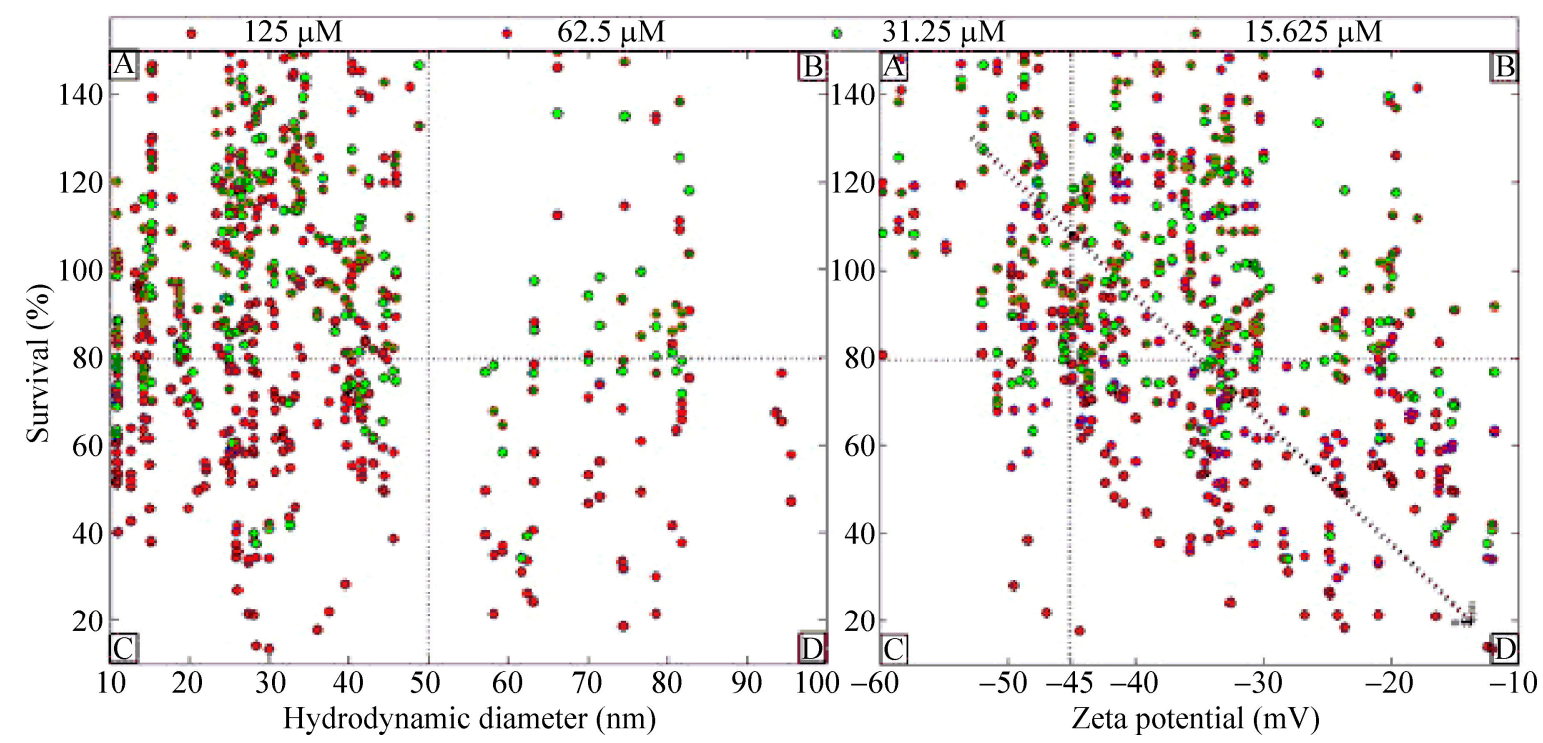

Figure 9 Cell survivability reported by Patra and Dasgupta [89], summarizing influences of particle size and $\zeta$ potential. Each point represents a mean percentage cell survival assessed in an experiment performed in triplicate for each given nanoparticle size (left panel) or for a given $\zeta$ potential (right panel). The colors of data points represent different concentrations of nanoparticles, as indicated at the top of the figure. Reproduced with permission from Ref. [89], (c) 2012 Elsevier Inc. 


\section{Gold nanorods}

AuNPs with a rod-like morphology (gold nanorods, AuNRs) are of particular interest, because of their anisotropic shape. Due to their non-spherical geometry, these particles have both a transverse and longitudinal plasmon [90]. The absorption profile includes 2 absorption bands: One due to light absorbed along the short axis (transverse) and the other due to absorption along the long axis (longitudinal). As the rod length increases, so does the longitudinal band red shift, along with an increase in the extinction coefficient.

Among different shapes, AuNRs have been reported to elicit higher toxicity than their spherical counterparts [21]. However, the mechanism of this elevated toxicity of AuNRs remains to be elucidated.

An interesting study was conducted by Takahashi et al. [85], who investigated the cytotoxicity of AuNRs in HeLa cells after $24 \mathrm{~h}$ of incubation. CTAB, a cationic micellar surfactant necessary for the preparation of AuNRs, was substituted by phosphatidylcholine (PC), and the resulting PC-NRs exhibited lower cytotoxicity than CTAB-NRs. Cell viabilities are presented in Fig. 10. In the case of PC-NR solutions, very little cytotoxicity was observed at concentrations up to $1.45 \mathrm{mM}$, with viability higher than $80 \%$. These results must be compared with the viabilities observed with CTAB-NR particles, where a dramatic decrease is observed with

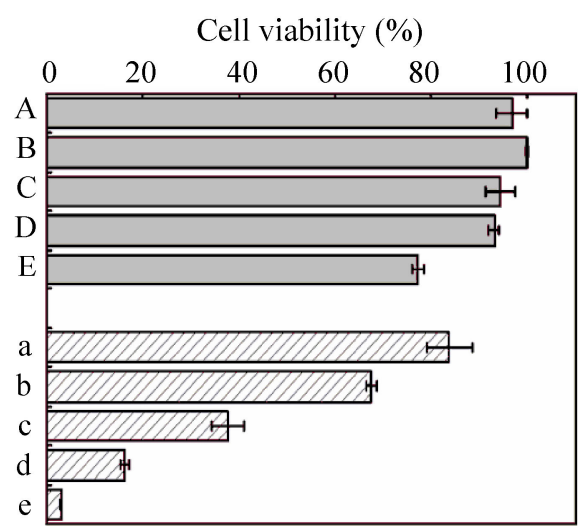

Figure 10 Viabilities of HeLa cells after incubation with PC-NR solutions (A-E) and twice-centrifuged CTAB-NR solutions (a-e). NR concentrations: $0.09 \mathrm{mM}(\mathrm{A}, \mathrm{a}), 0.18 \mathrm{mM}(\mathrm{B}, \mathrm{b}), 0.36 \mathrm{mM}$ (C, c), $0.72 \mathrm{mM}$ (D, d), and $1.45 \mathrm{mM}(\mathrm{E}, \mathrm{e}))$. Reproduced with permission from Ref. [85], (C) 2006 American Chemical Society. increasing particle concentrations. Since PC is not inherently toxic to living cells, Takahashi et al. [85] concluded that PC-NRs exhibit reduced cytotoxicity.

The same basic phenomenology occurs when CTAB is substituted by poly(acrylic acid) (PAA) or poly(allyamine hydrochloride) (PAH) polymers (molecular weight $15 \mathrm{kDa}$ ) [91]. Substitution with these polymers results in a significant reduction of cytotoxicity, with cell viability of a human colon cancer cell line (HT-29), measured by the MTT assay after 4 days of exposure to a particle concentration of $0.4 \mathrm{nM}$, increasing from $30 \%$ with CTAB-NRs to more than $90 \%$ with PAA-NRs and more than $80 \%$ with PAH-NRs.

It is worth nothing that, with the exposure to growth media with serum proteins (containing 10\% bovine serum albumin), the three types of AuNPs (AuNRs coated with CTAB, PAA, and PAH) present approximately the same $\zeta$ potential $(\sim-20 \mathrm{mV})$, i.e., the same effective surface charge, and have an effective size with diameters in the $30-40 \mathrm{~nm}$ range. These findings suggest that, at least with AuNRs, surface functionalization, rather than the size and surface charge, exerts a major influence on cytotoxicity.

Huff et al. [92] exposed KB cells to CTAB-coated AuNRs to examine their internalization by monitoring with two-photon luminescence (TPL) microscopy. CTAB-coated nanorods were found to localize near the perinuclear region within the $\mathrm{KB}$ cells. Cells appeared to be unaffected by the internalized AuNRs 5 years following exposure, since they grew to confluence over that period. This study, along with work by other groups, suggests that CTAB promotes nanorod uptake by cells, which could explain the cytotoxicity observed by Niidome et al. [84] with CTAB-stabilized nanorods.

In order to reduce the high cytotoxicity observed with CTAB-stabilized AuNRs, Huff et al. [92] developed PEG-modified AuNPs with a nearly neutral surface charge that exhibited little cytotoxicity in vitro. However, as pointed out by Khlebtsov and Dykman [24], the difficulties in assessing the toxicity of CTAB-coated AuNPs arise from the tendency of these particles to aggregate, inducing a release of $\mathrm{CTAB}$ into the surrounding medium, which can cause toxicity by itself. 
The influence of surface modifications of administered AuNRs by direct intravenous injection on adverse effects on blood vessels has been investigated by Alkilany et al. [93]. Surfactant-capped AuNRs were synthesized and either coated with a polyelectrolyte (PE) to prepare PE-AuNRs, or modified with thiolated PEG to prepare PEG-AuNRs. These authors demonstrated that therapeutic concentrations of PE-AuNRs, but not PEG-AuNRs, are toxic to the vascular endothelium, suggesting that differences in toxicity (as well as cellular uptake) between PE-AuNRs and PEG-AuNRs reflect the presence of free surfactant molecules and protein adsorption. Finally, the authors pointed out that toxic effects and cellular uptake into the vascular endothelium can elicit adverse effects following systemic administration of AuNRs, but could be prevented with appropriate surface functionalization.

In another study, Hauck et al. [86] produced nanorods $(18 \times 40 \mathrm{~nm}$ in size) exhibiting different surface charges through layer-by-layer coating with different polyelectrolytes, including CTAB, poly(4styrene sulfonic acid) (PSS1), poly(diallydimethyl ammonium chloride) (PDADMAC), PAH, and poly(4styrene sulfonic acid) (PSS2). Their $\zeta$ potentials varied from highly negative to highly positive (from -69.5 to $52.2 \mathrm{mV}$ ). The uptake of these nanorods by HeLa cervical cancer cells, together with their toxicity, was evaluated using a dye-exclusion cell viability assay, with results shown in Fig. 11. As shown, all investigated nanorod formulations, with the exception of CTAB-coated nanorods at the concentration of $150 \mu \mathrm{M}$ in serum-free medium, resulted in cell viability of approximately $95 \%$, which is not significantly different from the viability observed in control cells.

Taken together, these findings confirm that a high concentration of $\mathrm{CTAB}$, such as the one employed in the synthesis of nanorods, provokes some concerns regarding their toxicity $[94,95]$. The cytotoxicity of CTAB-coated AuNRs in serum-free media was confirmed by Hauck et al. [86], who also demonstrated that the viability of the cell was greatly increased in serum-containing media, reaching viability levels measured in control cells. This effect has been attributed to the adsorption of proteins to the surface of nanorods, reducing the CTAB cationic surface. In the case of nanospheres, however, recent studies have

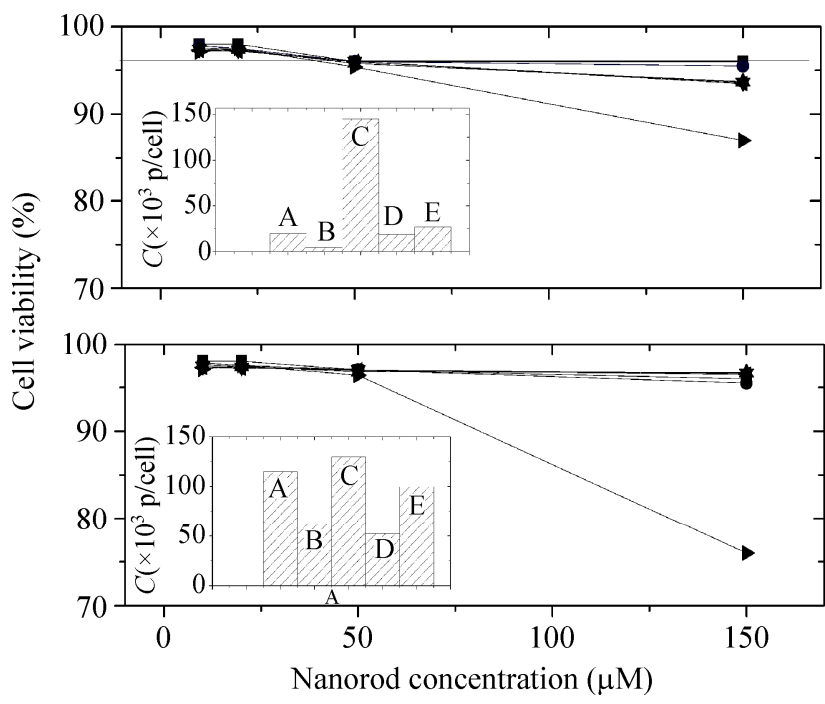

Figure 11 Toxicity of AuNRs in a medium containing fetal bovine serum (upper panel) and in serum-free medium (bottom panel), at different gold atom concentrations and with different surface coatings: PSS1 (•); PDADMAC (•); PHA (^); PSS2 (•); CTAB $(\bullet)$. The two insets show the cellular uptake of nanorods in media containing serum (upper panel) and without serum (bottom panel). CTAB (A); PSS1 (B); PDADMAC (C); PHA (D); PSS2 (E). Reproduced with permission from Ref. [86], (C) 2008 WILEY-VCH Verlag GmbH \& Co. KGaA, Weinheim.

shown that CTAB-coated AuNPs alone elicit minimal in vitro cytotoxicity if the surfactant concentration is reduced [7].

\section{Gold nanoshells}

Gold nanoshells are a class of nanoparticles composed of a silica dielectric core coated with an ultrathin metallic gold layer, displaying tunable optical resonances. This core/shell structure, with diameters ranging in size from 10 to $200 \mathrm{~nm}$, allows for the gold nanoshells to be made by varying the relative core and shell thicknesses, resulting in particles which either preferentially absorb or scatter light in the visible and near-infrared (NIR) regions of the spectrum.

As novel nanostructures, they possess a remarkable set of optical, chemical, and physical properties, which make them ideal candidates for applications in cancer detection, cancer treatment, cellular imaging, and medical biosensing.

A key investigation evaluating the toxicity of these nanoparticles was carried out by Hirsch et al. [96]. Incubation of human breast epithelial carcinoma 
SK-BR-3 cells (ATCC) with gold-silica nanoshells (core $55 \mathrm{~nm}$, shell $10 \mathrm{~nm}$ ) suspended in serum-free medium $\left(4.4 \times 19^{9}\right.$ particles $\left./ \mathrm{mL}\right)$ at the temperature of $37^{\circ} \mathrm{C} \mathrm{did}$ not affect cell viability, suggesting that therapy by itself is not cytotoxic.

Nanoshells with dimensions of approximately $130 \mathrm{~nm}$, providing peak optical scattering and absorption efficiencies in the NIR $(\sim 800 \mathrm{~nm})$, were produced by Loo et al. [97]. SKBr3 breast adenocarcinoma cells were added to the solution of nanoshells at a volumetric ratio of $1: 9$ and incubated for $1 \mathrm{~h}$. No difference in viability was observed between cells incubated with nanoshells and untreated control cells Nanoshells used in this work are silica-gold coreshell nanoparticles, with nominal core size of $110 \mathrm{~nm}$ and 10-nm thick shell [98]. In order to provide the steric repulsion needed to reduce the aggregation of particles in the blood and deter protein absorption, nanoshells were stabilized by coating with PEG.

Healthy female albino mice older than 6 weeks of age and with body mass of approximately $15 \mathrm{~g}$ were anesthetized with isoflurane and injected with $100 \mu \mathrm{L}$ of PEGylated nanoshells suspended in $0.9 \% \mathrm{NaCl}$ via the tail vein.

Although nanoshells were quickly scavenged from the blood after 28 days, elevated levels of gold were still present in the liver and spleen, with concentrations 3 orders of magnitude higher than the pretreatment levels. These elevated levels, however, were not associated with any physiological complications.

In a recent study, Khlebtsov et al. [24,99] investigated the toxic effects induced by PEG-coated silica/gold nanoshells administered intravenously to rats at $75,150,225$, and $300 \mathrm{mg} \cdot \mathrm{kg}^{-1}$ doses. Fifteen days after injection, some macroscopic changes in the liver and spleen, as well as multiple macrofocal effusions of blood, were observed with the highest doses (225 and $\left.300 \mathrm{mg} \cdot \mathrm{kg}^{-1}\right)$. However, these results should not be considered definitive, since the morphological changes were detected only in some of the treated groups. In the majority of treated rats, necrosis of hepatocytes with pyknosis of the nucleus and other histological modifications were observed, despite being absent in normal samples [99].

Gold nanoshell particles with an average diameter of approximately $30 \mathrm{~nm}$ consisting of a thin gold wall with a hollow interior have been synthesized by Melancon et al. [100] and employed in photothermal ablation therapy for the destruction of epidermal growth factor receptor. These particles, which display a strong resonance absorption peak tunable in the NIR region, did not show any observable effects on cell viability, likely due to the absence of a silica core.

$\mathrm{Au}_{3} \mathrm{Cu}_{1}$ (gold and copper) nanoshells exhibited a promising effect on magnetic resonance (MR) in in vitro MR images [101]. In assessing the potential uses in in vivo MR imaging, these agents enhanced the contrast of blood vessels, suggesting their potential for use in MR angiography as agents for delineating the blood-pool. $\mathrm{Au}_{3} \mathrm{Cu}_{1}$ nanoshells with an average diameter of approximately $50 \mathrm{~nm}$ and a shell thickness of approximately $6 \mathrm{~nm}$ have been prepared by Su et al. [101]. Measurements of $\zeta$ potential indicated that these hollow $\mathrm{Au}_{3} \mathrm{Cu}_{1}$ nanoparticles had a negative surface charge of $-18 \mathrm{mV}$. These particles could be further optimized to assemble with multilayer polyelectrolytes on their surfaces, forming nanocapsules.

$\mathrm{Su}$ et al. used a WST-1 assay on a Vero cell line (monkey kidney cell line) to measure the activity of mitochondrial dehydrogenase enzyme, which is known to be related to cell viability. Nanoshells were biocompatible at dosages between 0.1 and $10 \mu \mathrm{g} \cdot \mathrm{mL}^{-1}$, while cell survival decreased with increasing doses. At $200 \mu \mathrm{g} \cdot \mathrm{mL}^{-1}$, cell viability was reduced to approximately $15 \%$ after $24 \mathrm{~h}$ of treatment. Authors hypothesized that the reduced viability could be associated, at least in part, with the nanoshells occupying the space in culture wells normally available for cell growth.

\section{Gold nanocages}

Gold nanocages (AuNCs), a new class of nanoscale agents developed for applications in bio-imaging, photothermal therapy and controlled drug release, are hollow porous AuNPs with sizes in the range between 10 and $150 \mathrm{~nm}$ [102]. These particles, consisting of hollow interiors and porous walls, are characterized by extraordinarily large cross-sections for both absorption and scattering.

Due to their tunable surface plasmon resonance (SPR) peaks, the relatively simple synthetic method, 
and their hollow-porous structure, AuNCs represent a new class of nanoscale agents, which elicit a marked synergic effect in cancer treatment. Nanocages with sizes in the 40-50 nm range exhibit optimal cell uptake, since their SPR is in the optical window of biological tissue $(\sim 800 \mathrm{~nm})$ [103].

Nanocages have been employed as photothermal agents for the selective destruction of cancerous or diseased tissue and served as drug delivery vehicles for controlled localized release of particle-loaded drugs in response to external stimuli [102]. Upon NIR irradiation [104], the photothermal properties of AuNCs lead to a rapid rise in local temperature. This effect favors the uncapping of the thermally sensitive gatekeeper compounds, allowing the release of the contents of the cage.

However, serious issues regarding toxicity and, in some cases, particle stability, remain to be addressed.

Wang et al. [105] explored the in vivo biodistribution of ${ }^{198} \mathrm{Au}$-doped AuNCs in mice bearing EMT-6 tumors. AuNC with 33-nm edge length covered by PEG chains with 5,000 kDa molecular weight were intravenously injected into mice, and bioluminescence images were sequentially captured using the IVIS in vivo imaging system. This breast cancer model is known to exhibit accumulation of nanoparticles in the tumor through the enhanced permeability and retention (EPR) effect. PEGyated nanocages rapidly accumulated in the tumor following injection, with significant accumulation also observed in the spleen and liver. It is worth mentioning that no adverse reaction was observed at the administered doses $\left(1.7 \times 10^{12}\right.$ particles/mouse $)$ during all experiments.

AuNCs of different sizes and surface functionalities have been employed by a number of research groups in a range of biomedical applications. For example, Kim et al. [106] used AuNCs with an outer edge length of $46 \mathrm{~nm}$ and a wall thickness of $7 \mathrm{~nm}$ with surface functionalized by PEG in B16 melanomas in vivo as contrast agents for photoacoustic tomography. PEGyated AuNCs of similar characteristics (outer edge length of $48 \mathrm{~nm}$ and wall thickness of $3.5 \mathrm{~nm}$ ) were employed by Chen et al. [107] as photothermal therapy against human glioblastoma cell line in mice.

As another example, antibody-conjugated AuNCs were attached to the surface of cells through antibody-antigen binding [108] and then internalized into the cells by receptor-mediated endocytosis. No morphological changes or plasma membrane damage was observed. These studies highlight the novelty of the structures of these AuNPs and forecast exciting new perspectives in cancer therapy.

\section{Gold nanostars}

Gold nanostars (AuNSs), prototype anisotropic particles, have a tunable morphology, with the number and length of the branches determined during the synthesis procedure. AuNSs are characterized by unusual optical properties that render them particularly suitable for a variety of biomedical uses. Their peculiar shape provides a large surface area, which allows for loading of a high concentration of drug molecules, reducing the number of gold particles required and thereby reducing toxicity.

Only a very limited number of studies have evaluated AuNSs thus far, primarily because their use is greatly limited by the toxicity of CTAB and other surfactants employed in their formulation, Additionally, the particular morphology AuNSs favors the formation of aggregates [109], reducing their potential applications. A novel method for production of surfactant-free monodispersed AuNSs with an easy surface functionalization was recently proposed by Yuan et al. [110], allowing for the production of particles with great potential for diagnostic applications and reduced toxicity.

A randomly branched gold nanostructure with a core size of $26-220 \mathrm{~nm}$ and a branch length of $8-114 \mathrm{~nm}$ have been recently produced by Trigari et al. [111], with extinction spectra that can be tuned from visible up to $1,500 \mathrm{~nm}$.

Star-shaped AuNPs with average width of $180 \mathrm{~nm}$ have been recently synthesized with $70 \%$ yield by Salinas et al. [112] and applied to mouse hippocampal slices during the recording of action potential activity of neurons in the CA3 area. While the firing rate markedly increased, no adverse effects on neuronal function were observed, supporting the feasibility of using AuNSs for applications in neurobiology.

The biocompatibility of PEGylated AuNSs (and bipyramides, too) incubated with melanoma B16-F10 
cells has been investigated by Navarro et al. [113], with cellular uptake assessed by dark-field microscopy. These particles were revealed to be rather monodisperse, with a tip-to-tip distance of $130 \mathrm{~nm}$, a spherical core of approximately $60 \mathrm{~nm}$, and approximately 10 branches per gold core. The authors [113] were able to remove $\mathrm{CTAB}$ and myristyl bromide, replacing the double layer surrounding the particles with PEG, a biocompatible polymer. Taking advantage of their peculiar shape and the resulting specific photo-physical properties, these particles can be easily detected within the cell, making them suitable agents for bioimaging applications.

The increased loading density of AuNSs, favored by their asymmetric shape, provides a simple means for improving their uptake in cancer cells. Dam et al. [114] demonstrated that a dense packing of DNA aptamer drug AS1411 (Apt) on AuNSs with sizes of $37 \mathrm{~nm}$ favors the internalization of these nanoconstructs in a wide range of cancer cells. These particles were taken up by pancreatic cancer cells and fibrosarcoma cells at a faster rate, resulting in increased death of cancer cells. Interestingly, treatments with these nanoconstructs had no adverse effects on normal cell lines [115].

The cytotoxicity of irregularly shaped urchin AuNPs with an average volume equal to that of a sphere $77 \mathrm{~nm}$ in diameter was investigated by Hutter et al. [116] in a microglial (N9) cell line, comparing the results with those obtained with nanospheres and nanorods covered with the same surface coating (either CTAB and PEG); rod and urchin AuNPs were relatively innocuous, in contrast to spherical particles.

Finally, the biodistribution of AuNSs (56 nm in size) in mouse liver, spleen, and blood vessels was investigated by Li et al. [117] using a quantitative photoacustic microscopy technique. Results demonstrated that AuNSs accumulated preferentially in the liver from blood circulation, with moderate associated toxicity.

\section{Towards a standardized protocol: Opportunities and recommendations}

The goal of toxicity assessment is to relate the physico-chemical properties of AuNPs to their toxicity, thereby facilitating the prediction of potential risks associated with their biomedical applications in humans, or at least providing insight into the optimal design, which would result in minimal toxicity. A review [118] dealing with this important topic was recently published, attempting to correlate parameters such as size, shape, charge, stability, material concentration, and ability to adsorb biological compounds with toxicity. Unfortunately, the authors of this review [118] report that, based on currently available data, the correlations of physico-chemical parameters with the whole toxicity is not straightforward. In view of the few examples cited in previous sections, it is obvious that cytotoxicity of AuNPs remains an open question and that there is an urgent need for standardization of the protocols employed in its evaluation. Johnston et al. [30] reviewed some perspectives on the need for a standardization of experimental protocols, including model species and cell types used and the methods of particle administration. More recently, a comprehensive analysis of potential adverse effects of nanomaterials, based on the findings of the PARTICLE_RISK research project funded by the European Commission, was presented by Johnston et al. [119]. These authors analyzed the physic-chemical characteristics of nanomaterials from a general point of view and identified possible attributes of nanomaterials that may be responsible for the observed toxic effects. These aspects include nanomaterial selection, dispersion of nanomaterials, their relevant doses and concentrations, identification of relevant cellular models, target sites, and nanomaterial distribution following exposure. In their review, the authors [119] propose potential solutions to overcome the uncertainties in the estimates of risks posed by nanomaterials. Starting from this analysis, we have applied their proposals to AuNPs.

Below, we will consider separately different parameters that have been experimentally proven to influence the toxicity of AuNPs. For each parameter, we will briefly discuss key aspects that need to be taken into account to progress towards a standardized protocol.

\subsection{Nanoparticle characterization}

Shape and size of nanoparticles are generally determined by two common techniques: Dynamic light 
scattering (DLS) and electron microscopy, including SEM and TEM. However, different artifacts may arise with these analyses, since TEM and SEM are performed under vacuum conditions. Additionally, DLS analysis of the autocorrelation function in highly polydisperse systems is not a trivial task, since DLS tends to overestimate mean particle size due to the high scattering intensity of larger objects, making the data difficult to interpret. To overcome these limitations and facilitate the imaging of samples in aqueous environments, cryogenic temperature electron microscopy (cryo-EM) should be used.

The surface charge of nanoparticles can be deduced from the measurement of the $\zeta$ potential using the laser Doppler electrophoresis technique. Measured values are generally affected by different factors, such as the $\mathrm{pH}$ of the solution, electrical conductivity (reflecting the thickness of the double layer and the concentration of particles present in the solution), and the aggregation effects that occur in solution. Since $\zeta$ potential can be strongly influenced by the surrounding medium, an accurate characterization of the sample under investigation is highly recommended. In this respect, notable attempt was made by the International Alliance for NanoEHS Harmonization (IANH) to carry out an inter-laboratory comparison of measurements of nanoparticle size and surface charge prior to biological impact assessment [120].

Another significant factor that needs to be considered is the fact that, largely, chemical and biological activities of nanoparticles are determined by the atoms on the particle surface. Since the ratio of surface to total atoms increases exponentially with decreasing particle size, a direct correlation between particle size and toxicity can be misleading, and caution should be exercised in the interpretation of the data [121].

Traces of impurities within the nanomaterial formulation may lead to additional toxic effects. For example, as reported above, the presence of free $\mathrm{CTAB}$ in solution, derived from the formulation of nanorods, may cause toxic effects in human colon carcinoma cells [91].

Often, modifications of AuNP surfaces through coating with polyelectrolytes or proteins can cause undesirable ionic interactions with biological systems [122], which can play a significant role in their toxicity.
As pointed out by Kong et al. [123], these surfacebound molecules can greatly influence the interactions between cells and nanoparticles, giving rise to unintended toxic effects, which are unrelated to the nanoparticles.

Finally, when aggregated, AuNPs show modified surface charges, which influence the cellular environment, altering cellular behavior and potentially resulting in cytotoxicity [124].

\subsection{Nanoparticle concentration and effective dose}

Nanoparticles tend to aggregate in different dispersion media and particularly in biological media, which exhibit a relatively high ionic conductivity, leading to the screening of the nanoparticle surface charge. Nanoparticles may aggregate in cell culture media due to the presence of ions and proteins. Ions can decrease the screening length of charged chemical groups at the particle surface, while proteins from the medium can cause replace surface-associated molecules in a thermodynamically favored process [125]. The formation of particle clusters prior to the interaction with cells or during the adsorption on the cell membrane provides the mechanism, which at least in part, accounts for the observed size-dependent particle uptake behavior. These issues demonstrate the need for real-time single-particle techniques to improve our understanding of the effects of particle clustering.

The extent of the clustering effect depends on the nanoparticle size, shape, charge, and the viscosity and density of solutions. This means that the effective number of nanoparticles, which interact with cells, remains largely undefined in most cases. As pointed out by Elsaesser et al. [126], there is considerable debate on which metric would be the most appropriate [127]. A quantitative interpretation of data requires three primary physical metrics: Particle mass, surface area, and particle number [128]. A consensus has been reached on the dose metric related to the number of particles per each cell or each sub-cellular compartment, even if this number can be difficult to estimate [129]. However, a dissenting opinion has been expressed by Wittmaack [130], who considers particle number per unit volume to be the optimal dose metric. 
In some circumstances, the total surface of nanoparticles may be a preferable metric, particularly when chemical reactions occurring at the particle surface play a dominant role. In this case, particle size and shape need to be known, since mass alone cannot predict the total surface area. In line with this opinion, a measure of surface area per unit volume is preferred by Joris et al. [131], since this metric would comprise both particle size and particle number. Additionally, the toxicological response is known to depend on the surface properties of nanoparticles and surface area exponentially increases with decreasing particle size.

In this context, it should be noted that a significant correlation was observed between the in vitro oxidative response and, the in vivo inflammatory response for different nanoparticles with distinct physicochemical properties when particle concentration was expressed in terms of surface area per unit volume [127, 132].

Consequently, it is desirable to obtain a measurement of the number of particles per cell using analytical tools such as liquid chromatography-mass spectrometry (LC-MS) and radioactive isotopes [133].

The levels of gold in tissues can also be determined using ICP-MS, providing a sensitivity of approximately $1 \mathrm{ng} \cdot \mathrm{kg}^{-1}$ of animal body weight [134]. Finally, for the localization of AuNPs in tissues, histology in combination with SEM and TEM techniques can be applied [135].

As pointed out by Rivera-Gil et al. [118] in relation to AuNPs, the entity whose toxicity is to be ascertained is a hybrid object, consisting of an inorganic core (gold in this case) and an organic coating. Since larger surface area is generally accompanied by higher chemical reactivity, greater attention should be paid to the surface area of the particles rather than to the properties of the core. However, it is illogical to state that toxicity can be attributed exclusively to the surface coating, or, conversely, to the metallic core. In support of this assertion, the two examples reported by Rivera-Gil et al. [118] should be noted. The first example discusses CTAB-capped AuNPs developed by Qiu et al. [136], which exhibit a toxicity that is a result of the CTAB shell and not the gold core. The second example deals with nanoparticles with different cores (gold, FePt), but with the same surface coating (a carbon-terminated polymer). In this case, cytotoxic effects are attributed to the unique properties of the inorganic core, rather than to the surface coating [137].

Comparisons between the results of different studies are complicated by the use of wide ranges of nanoparticle concentrations. As suggested by Johnston et al. [30], it would be useful to introduce the use of threshold doses such as the "no observed adverse effect level" (NOAEL) concentration into the analysis of toxicity. These threshold doses may be relevant for separating the toxic behavior at low concentrations, where AuNPs may mediate protective responses, from the behavior at higher concentrations, where toxic responses become evident.

Particle concentrations are frequently expressed as mass per unit volume $\left(\mu \mathrm{g} \cdot \mathrm{mL}^{-1}\right)$ to indicate the amount of nanomaterial, which interacts with the cells. However, this can result in significant differences between the particle number concentration and total surface area, with discrepancies of up to several orders of magnitude [138]. In fact, smaller diameter of spherical particles results in a greater increase in the surface-to-volume ratio. In Fig. 12, the behavior of the particle number concentration $C_{N}$ and the total surface concentration $C_{S}$ is presented as a function of nanoparticle size for a fixed nanoparticle concentration, expressed as mass per unit volume. As seen from the presented data, these quantities vary rather dramatically depending on the particle radius. As toxic effects are expected to be associated with the available surface area [139], this relationship can lead to an undefined effective concentration, rendering the particle number practically meaningless.

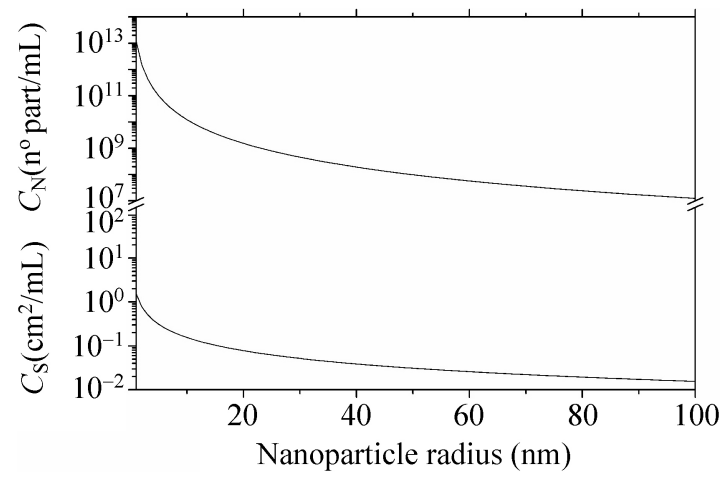

Figure 12 The numerical particle concentration $C_{\mathrm{N}}$ (upper curve) and total area concentration $C_{\mathrm{S}}$ (bottom curve) as a function of the nanoparticle radius $R$ for a given value of the mass concentration $C=1 \mu \mathrm{g} \cdot \mathrm{mL}^{-1}$. 
To overcome this difficulty, the concentration of particles introduced into the cell cultures in cytotoxicity assay should be expressed in terms of mass per unit surface area of the culture dish $\left(\mu \mathrm{g} \cdot \mathrm{cm}^{-2}\right)$ or in terms of mass per cell numbers $\left(\mu \mathrm{g} / 10^{6}\right.$ cells), instead of commonly used mass per unit volume (e.g., $\mu \mathrm{g} \cdot \mathrm{mL}^{-1}$ ) or number of nanoparticles per unit volume.

\subsection{Stablity of AuNPs}

Nanoparticle stability is dependent on the organic coating, which imparts electrostatic or steric repulsion. As we have stated above, the protein corona plays an important role in nanoparticle stability. However, its presence also contributes to making the characterization of AuNPs complicated in biological systems. As described by Monopoli et al. [61] with $\mathrm{SiO}_{2}$ nanoparticles, the protein corona at protein concentration appropriate for in vitro cell studies is different from the corona at concentrations encountered in in vivo studies. This discrepancy suggests further complications in the interpretation and comparison of different experimental results.

\subsection{Cellular uptake}

In in vivo experiments, the size and shape of the nanoparticles should be chosen with care, since cellular uptake, nanoparticle kinetics, and internalization are strongly dependent on the particle size. Moreover, in addition to the specific cell-nanoparticle interactions, researchers must take into account the fact that large particles, which can be detected by the immune system, will be rapidly removed and delivered to the liver and the spleen, while, conversely, very small nanoparticles will be easily excreted through the kidney by renal filtration. As suggested by Rivere-Gil et al. [118], the optimal particle size is in the range between 10 and $100 \mathrm{~nm}$.

We have already mentioned the uptake by HeLa cells of spherical and rod-shaped AuNPs of different sizes, investigated by Chithrani et al. [64]. This study suggested that cellular uptake was mediated by the adsorption of serum proteins onto the particle surface, favoring particle entry via clathrin-mediated endocytosis. This proposed relationship is largely supported by the observation that this active uptake mechanism is greatly reduced at low temperature (i.e., $4{ }^{\circ} \mathrm{C}$ ), where adenosine triphosphate (ATP) generation is reduced. These key findings, along with the proposed hypothesis that the saturation of nanoparticle uptake results from the extent of protein binding, were subsequently confirmed by Chithrani and Chan [140] in their investigation of the uptake of transferrin-coated nanoparticles by epithelial, fibroblast, and neuronal cell lines. These studies confirmed the involvement of endocytosis in nanoparticle uptake and highlight the importance of protein adsorption. However, in addition to uptake induced by active mechanisms such as endocytosis, particle entry into the cells may also occur by simple diffusion (passive mechanisms).

Electrical surface charge and particle hydrophobicity are two key parameters determining cellular uptake of functionalized AuNPs. Whereas interactions between cationic nanoparticles and negatively charged groups at the cell membrane resulting from the presence of sialic acid are easily understood based on electrostatic attraction, the role of hydrophobicity in this process is less clear.

The importance of the chemical structure of nanoparticle surface functional groups has been highlighted by Green et al. [141] who employed nanoparticles coated with cell-penetrating peptides, such as those containing the amino acid sequence Arg-Gly-Asp. These structures were found to exhibit favorable characteristics, including near-neutral $\zeta$ potential and acceptable stability in serum. A more complex structure was used by Kanduc et al. [18], who demonstrated that penetration into the nucleus after endosomal escape occurs with AuNPs coated with PEG and those conjugated with peptides containing the Arg-Gly-Asp sequence.

A general scheme illustrating the routes of uptake and translocation of nanoparticles, including AuNPs and other particles, is shown in Fig. 13.

Further in vivo studies are needed to elucidate the pathways and cellular entry routes of AuNPs in complex subcellular organisms [126].

\subsection{Cytotoxicity assay}

Various assays have been used to study the toxic effects of nanoparticles in cell cultures, depending on the cellular parameter of interest. Viability assays were 


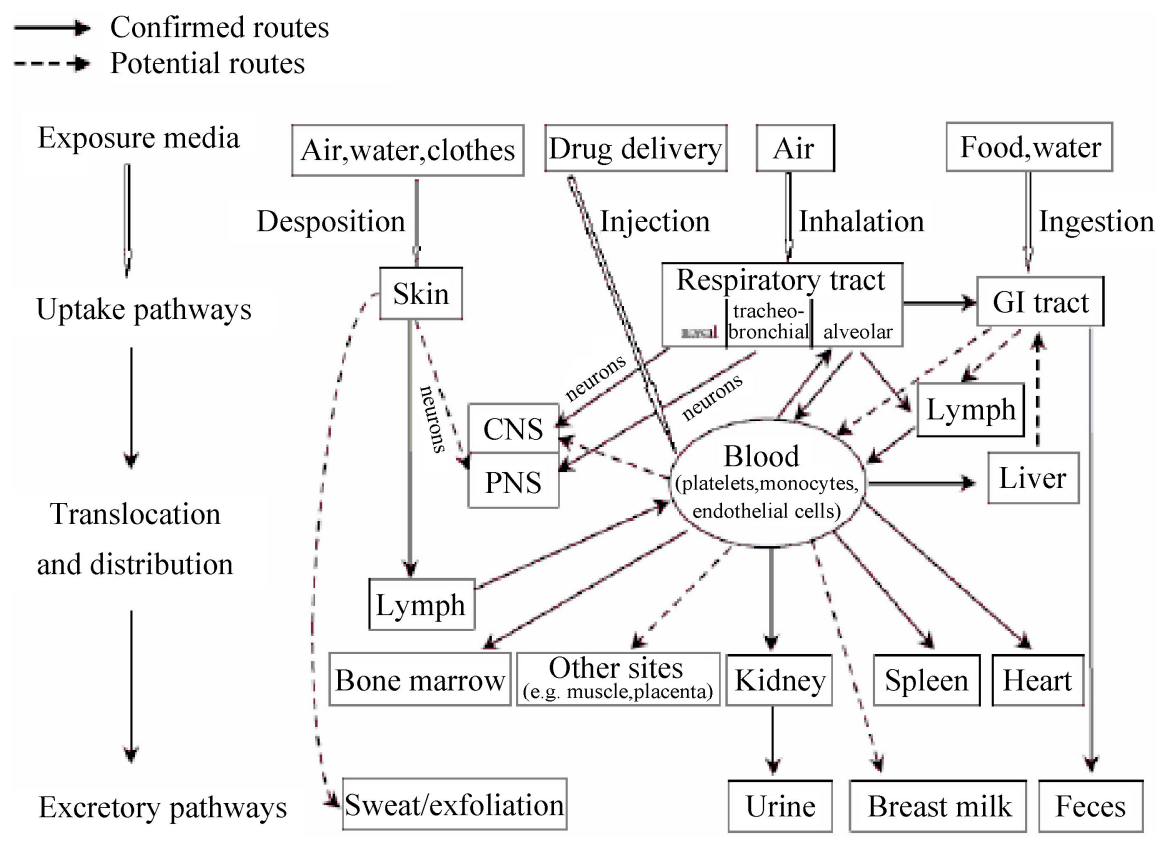

Figure 13 A general scheme depicting the biokinetics of nanoarticles, including uptake and translocation routes already ascertained (full arrow) and routes that are hypothetical at this time (dotted arrow). Reproduced with permission from Ref. [121], (C) 2005 Environmental Health Perspectives.

used to assess the overall dose-dependent toxicity in cultured cells following exposure to nanoparticles [142]. These methods include LDH leakage assay, trypan blue or PI assays, 4-[3-(4-iodophenyl)-2-(4nitrophenyl)-2H-5-tetrazolio]-1,3-benzene disulfonate (WST-1) or MTT assays, calcein AM assay, and measurements of fluorescent Annexin V or caspase substrates. LDH assay measures the release of the enzyme into the culture medium as an indicator of the cell membrane disruption, while trypan blue and PI assays are dye-exclusion techniques in which the uptake of the dye into the cell can be used as an index of cell membrane permeability. MTT assay, technically similar to the WST-1 assay, measures the enzymatic activity of cellular mitochondria and is considered the "gold standard" for in vitro toxicology studies. Finally, calcein AM assay measures intracellular esterase activity, while assays measuring Annexin V and caspase substrates can be used as indicators of apoptosis.

Cell viability is a somewhat general term. Since each of the previously listed methods aims to determine one or more of the cellular parameters, their findings cannot be compared directly between the assays, since they measure different parameters [143]. As a general guideline, cytotoxicity should be verified with at least two independent assays to avoid any misinterpretations of results.

A further problem concerns the occurrence of false-positive and false-negative results, making crosschecking of the data with alternative independent assays to ensure reliability of the results desirable, helping to avoid errors. As an illustrative example, NPs with particular optical properties can alter the results of assays based on spectrophotometric measurements, while NPs with high absorbance capacity and catalytic activity may interfere with enzymes or assay substrates $[144,145]$. Another example would be the case of AuNP-treated cells, in which dead cells are imaged with the commonly used fluorescent PI dye. Normally, fluorescent PI molecules cannot penetrate the cell membrane and enter the cell. However, in a number of experiments PI was observed to enter the cells in parallel with the endocytosis of nanospheres, resulting in a false-positive toxicity result [36].

Immortalized cell lines, like HeLa cells, are commonly used to compare the cytotoxicity of nanoparticles with varying sizes and surface chemistries (as an example, please see Fig. 8). In using these cell 
lines, one must take into consideration that both the genome and proliferation pattern of immortalized cell lines deviate from those of normal healthy cells. Cells in the logarithmic growth phase are more sensitive to toxic effects than those in the stationary phase [34].

As a final comment, it should be noted that there is often a lack of correlation between in vivo and in vitro results, indicating that the design of better assays with physiologically relevant end-points must be intensely pursued.

\section{6 "Toxicity factor"}

A publication by Pompa et al. [19] introduced a systematic evaluation of AuNP toxicity by means of a representation of nanotoxicology as a multiparametric space, in which different variables are related to the chemico-physical characteristics of the nanoparticles (composition, shape, surface chemistry, and surface charge). Using this approach, the authors were able to elucidate whether size-dependent toxic effects observed in vivo are due to the dimensions of the nanoparticles, their exposed surface area, or the total number of NPs taken up.

After defining a dose-response curve for AuNPs ingestion in the Drosophila melanogaster model system, Pompa et al. [19] characterized specific types of nanoparticles using a "toxicity factor" based on specific parameters derived from the mathematical fitting of the toxicity observed in the model organism, thereby defining a universal toxicity scale on which nanomaterials range from highly toxic to biocompatible nanomaterials (Fig. 14).

A more general three-dimensional phase diagram of toxicity based on three independent variables such as particle size, $\zeta$ potential, and dispersibility (hydrophobicity) is presented in Fig. 15. In this case, data were obtained from qualitative biocompatibility trends determined by in vivo screening of approximately 130 nanoparticles, including AuNPs, fullerenes, metal oxides, polymers, liposomes, dendrimers, and quantum dots [146]. This diagram demonstrates a number of general trends which are common to all nanoparticles investigated. For example, cationic particles are more likely to be toxic than larger, relatively hydrophobic, particles, which can be easily removed by the reticuloendothelial system. Particles

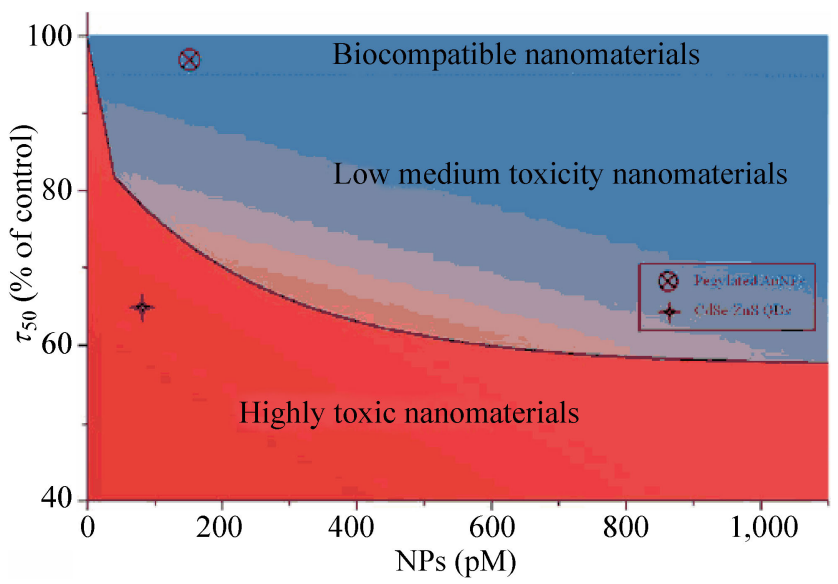

Figure 14 Schematic representation of toxicity regions of different nanomaterials (highly toxic, low/medium toxicity, biocompatible). The black line represents the reference toxicity curve (bi-exponential fit) of 15-nm citrate-capped AuNPs. The toxicity levels of PEGylated AuNPs (150 pM) and quantum dots with carboxyl terminals (85 pM) are also presented. Reproduced with permission from Ref. [9], (C) 2011 Royal Society of Chemistry.

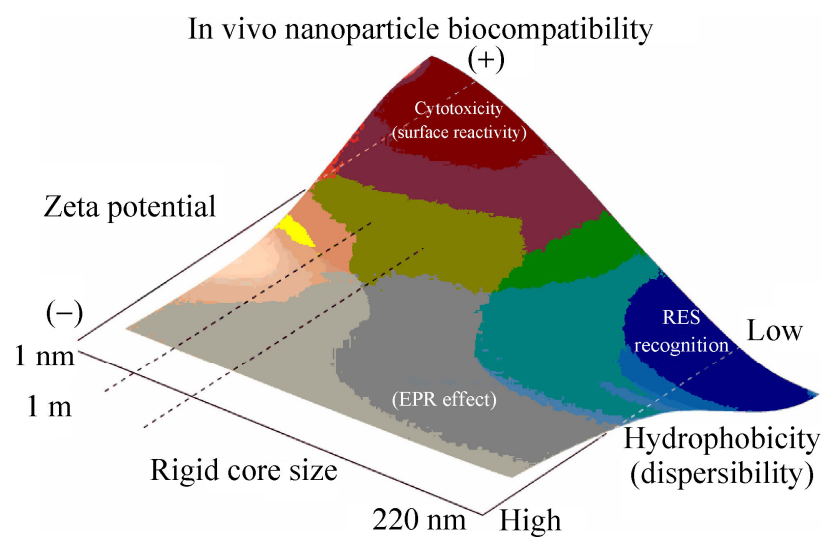

Figure 15 Biocompatibility three-dimensional phase diagram plotting the results of in vivo screening of different nanoparticles employed for therapeutic purposes. The independent variables are particle size, $\zeta$ potential, and dispersibility (particularly the effect of hydrophobicity). Bio-compatibility is reflected in the color of data points, with red representing likely toxicity, blue likely safety, and blue-green-yellow intermediate levels of safety. Reproduced with permission from Ref. [146], (C) 2009 Nature Publishing Group.

of mid-range sizes and relatively neutral surface charges exhibit enhanced permeation and retention effects.

\subsection{Gold nanoparticle-membrane interactions}

A final comment is in order. Interactions of nanoparticles with the cell membrane and their behavior 
in the proximity of membrane surfaces influence the mechanisms by which nanoparticles attach to the membrane itself, which in turn is the initial step in the process that leads to cytotoxicity. Since both biological membranes and nanoparticles bear a surface charge, electric double layer and van der Waals interactions are of preeminent importance. The importance of electrostatic interactions between charged AuNPs and phospholipid monolayers used as model membranes has recently been emphasized in a publication by Torrano et al. [147], who investigated the behavior of nanoparticles bearing different charges by studying negatively charged particles coated with citrate anions or positively charged particles functionalized with cationic poly(allylamine hydrochloride). Additionally, hydration and undulation forces originating from dynamic fluctuations of the membrane need to be considered as well.

Because of the complexity of the phenomenology involved in the behavior of biological membranes, it may be suitable to employ model membranes with known composition. This experimental approach would, at least in principle, allow a systematic investigation of the key parameters that control the attachment of nanoparticles to membranes. Relatively few such studies have been carried out to date, with the analyses of this problem by Nel et al. [146] and Negoda et al. [148] deserving special mention. More recently, a critical insight into these mechanisms was provided by Chen and Bothun [149].

\subsection{Effects of defects and impurities}

It should be noted that the inevitable presence of defects and impurities in particle formulations, favored by smaller particle size and the accompanying increase in the surface-to-volume ratio, was found to alter nanoparticle toxicity. Podila and Brown [150] have recently discussed this important, and frequently neglected, aspect.

The presence of surface defects including topological defects, vacancies, dislocations, grain boundaries, and different surface states may alter interactions between nanoparticles and cells or subcellular structures to a different extent, eliciting cytotoxic effects.

While these effects have been studied with a variety of nanostrucures and differently engineered materials, less attention has been directed to AuNPs. For example, the electronic properties of insulator and semiconductors monooxides $\mathrm{SiO}_{2}$ and $\mathrm{CuO}$, respectively) were shown to play an important role in cytotoxicity, as recently demonstrated by $\mathrm{Xu}$ et al. [151]. By intentionally introducing structural defects in multi-walled carbon nanotubes, Fenoglio et al. [152] have demonstrated that particles with structural defects can induce acute toxicity in lungs.

The influence of structural defects on AuNP toxicity remains to be thoroughly explored. A possible interrelation between different causes of cytotoxic effects, including structural defects, is presented in Fig. 16.

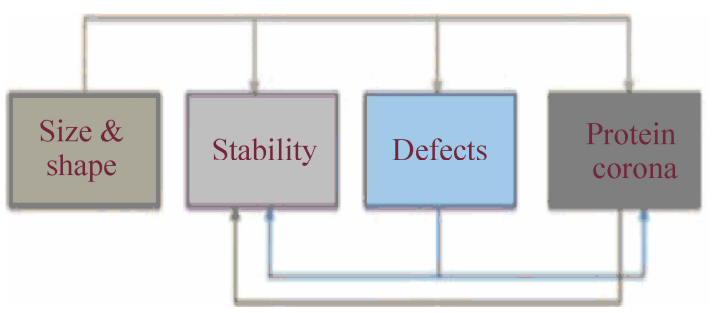

Figure 16 Schematic representation of how interrelations between particle size, shape, and defects determine the protein corona, resulting in particles which elicit different cellular or subcellular effects, potentially manifesting as a cytotoxic response. Reproduced with permission from Ref. [150], (C) 2012 Wiley Periodicals, Inc.

\section{Conclusions and outlook}

The general opinion that AuNPs are non-toxic, based on widespread perception of bulk gold as biocompatible, is becoming increasingly disputable. In consideration of the rapid growth in the use of AuNPs in diverse biomedical applications, recent studies have raised concerns regarding the toxicity of gold when used in nano-sized particles. The potential toxicity of AuNPs appears to be multi-faceted and difficult to predict [42]. Additionally, the lack of a common toxicity database hinders comparisons between results of different research groups.

Despite the promising future of AuNPs in various biomedical fields, there are a number of fundamental issues that remain to be addressed, including the questions concerning the therapeutic doses. More importantly, it is mandatory to define the therapeutic window within which nanoparticles can be employed 
without eliciting any side-effects. Furthermore, nanoparticles must be rigorously purified, with control of purity extended to each reagent employed in the production of AuNPs. Relevant questions deal with the long-term fate of AuNPs in the organism and the long-term effects of exposure to nanoparticles, which need to be thoroughly investigated in the widest possible scenario before AuNPs can be used in humans without concerns about health risks. In this respect, it is also important to note the differences between toxicity (cytotoxicity) and cellular damage, which need to be further clarified. Nanoparticles that elicit little or no cytotoxicity, as ascertained using standard assays, may cause cellular damage. As an illustrative example, Perdonet et al. [62] found that citrate-capped AuNPs with diameters of $13 \mathrm{~nm}$ induced the formation of actin filaments, resulting in a decrease in cell proliferation, adhesion, and motility, while they were observed to elicit no toxicity in skin cells.

Another point that warrants attention is the possibility that in vitro investigations may not be sufficiently representative of in vivo effects. The need for more reliable in vitro models with a high predictive power was recently discussed by Joris et al. [131]. As emphasized by Oberdorster et al. [121], the lack of definitive toxicology data does not currently allow for an adequate risk assessment and the precautionary principle should not be used in view of the potential hazards of AuNPs when administrated intentionally for improving human health.

Based on this scenario, there is a well-supported need to investigate the toxicological impact of AuNP and develop early indicators that could detect possible adverse health effects. At present, the conflicting data in the literature regarding AuNP bioactivity, reflecting differences in the laboratory research protocols, makes it difficult to evaluate and generalize important aspects of their biological effects and does not allow us to reach a definitive conclusion and a consensus opinion regarding AuNP cytotoxicity.

In this review, we have selected a number of relevant studies dealing with both in vivo and in vitro cytotoxicity of AuNPs from recent literature, with the aim of highlighting the urgent need to develop a common platform for investigating the extent of toxicity in different experimental frameworks.

Coordination between different research groups to establish the proper correlation between the parameters describing the physico-chemical properties of AuNPs and their effects on biological structures of different complexity is greatly desirable. This coordination can be attained only if standardization is introduced in all steps involved in the analysis of toxicity, beginning with the characterization of nanoparticles (particle size, shape, charge, and surface functionalization) and the experimental protocol (animal model, organs, tissues, and cells), up to the choice of the method for assessing the toxicity.

It would be very desirable to develop a predictive paradigm that could start from the physico-chemical properties of AuNPs and, based on the results of in vitro experiments, lead to predictions about their possible physiological effects in vivo.

This approach has been attempted at the University of California's Centre for Environmental Implications of Nanotechnology (UC CEIN) and the UCLA Centre for Nanobiology and Predictive Toxicology (CNPT) [153, 154], as recently reviewed by Sun et al. [155].

In light of the experimental work performed thus far, the use of a standardized experimental protocol is critical to provide a more rigorous evaluation of AuNP bio-compatibility versus cytotoxicity and to obtain reliable and realistic data. In the last part of this review, we highlight the most significant factors that need to be taken into consideration and summarize the critical issues that should be addressed when designing experimental protocols for the assessment of AuNP toxicity.

Even if not directly connected to the problem of toxicity, the studies on the properties of oligonucleotidemodified AuNP conjugates that can play a relevant role as intracellular gene regulation agents are of considerably interest, since they open new possibilities in the development of therapeutic and gene delivery systems. For example, Kim et al. [156] found that AuNPs functionalized with covalently attached oligonucleotides activate immune-related genes and pathways in human peripheral blood mononuclear cells. Moreover, transcription factor-based gene regulation is a promising approach for many biological applications. Additionally, AuNPs functionalized 
with functional peptides, can perform some of the functions of natural transcriptional factors [157], such as program stem cells to create specific tissues or, even more interestingly, to revert the cells back to an earlier developmental state. Finally, cancer is regulated by a number of signaling pathways and the determination of protein expression provides a way to study the mechanism of tumor progression [158]. Using AuNPs functionalized with monolayer of hairpin DNA with a specific sequence for the key protein mRNA, Xue et al. [159] detected the expression of STAT5B, AKT, and mTOR genes in living cancer cells. These examples demonstrate that interactions of multifunctional AuNPs with mammalian cells are among the hottest areas in current biomedical research.

\section{Acknowledgements}

We acknowledge support by grants from Ateneo Sapienza 2014/C26A14FCZP and 2013/C26A13HRZ4. This work has been partially supported by the Dipartimento di Chimica, "Sapienza" Universita' di Roma through the "Supporting Research Initiative 2013".

\section{References}

[1] Timbrell, J. A. Biomarkers in toxicology. Toxicology 1998, 129, 1-12.

[2] Schmid, O.; Möller, W.; Semmler-Behnke, M.; Ferron, G. A.; Karg, E.; Lipka, J.; Schulz, H.; Kreyling, W. G.; Stoeger, $\mathrm{T}$. Dosimetry and toxicology of inhaled ultrafine particles. Biomarkers 2009, 14, 67-73.

[3] Grass, R. N.; Limbach, L. K.; Athanassiou, E. K.; Stark, W. J. Exposure of aerosols and nanoparticle dispersions to in vitro cell cultures: A review on the dose relevance of size, mass, surface and concentration. J. Aerosol Sci. 2010, 41, 11231142.

[4] Ghosh, P.; Han, G.; De, M.; Kim, C. K.; Rotello, V. M. Gold nanoparticles in delivery applications. Adv. Drug. Delivery Rev. 2008, 60, 1307-1315.

[5] Eck, W.; Nicholson, A. I.; Zentgraf, H.; Semler, W.; Bartling S. N. Anti-cd4-targeted gold nanoparticles induce specific contrast enhancement on peripheral lymphonodes in x-ray computed tomography in live mice. Nano Lett. 2010, 10, 2318-2322.
[6] Bhattacharya, R.; Mukherjee, P. Biological properties of “naked” metal nanoparticles. Adv. Drug. Delivery Rev. 2008, 60, 1289-1306.

[7] Connor, E. E.; Mwamuka, J.; Gole, A.; Murphy, C. J.; Wyatt, M. D. Gold nanoparticles are taken up by human cells but do not cause acute cytotoxicity. Small 2005, 1, 325-327.

[8] Dobrovolskaia, M. A.; McNeil, S. E. Immunological properties of engineered nanomaterials. Nat. Nanotechnol. 2007, 2, 469-478.

[9] Patra, H. K.; Banerjee, S.; Chaudhuri, U.; Lahiri, P.; Dasgupta, A. K. Cell selective response to gold nanoparticles. Nanomedicine 2007, 3, 111-119.

[10] Peng, G.; Tisch, U.; Adams, O.; Hakim, M.; Shehada, N.; Broza, Y. Y.; Billan, S.; Abdah-Bortnyad, R.; Kuten, R.; Haick, H. Diagnosing lung cancer in exhaled breath using gold nanoparticles. Nat. Nanotechnol. 2009, 4, 669-673.

[11] Pan, Y.; Neuss, S.; Leifert, A.; Fischler, M.; Wen, F.; Simon, U.; Schmid, G.; Brandau, W.; Jahnen-Dechent, W. Sizedependent cytotoxicity of gold nanoparticles. Small 2007, 3, 1941-1949.

[12] Zhang, X. D.; Guo, M. L.; Wu, H. Y.; Sun, Y. M.; Ding, Y. Q.; Feng, X.; Zhang, L. A. Irradiation stability and cytotoxicity of gold nanoparticles for radiotherapy. Int. J. Nanomedicine 2009, 4, 165-173.

[13] Sung, J. H.; Ji, J. H.; Park, J. D.; Song, M. Y.; Song, K. S.; Ryu, H. R.; Yoon, J. U.; Jeon, K. S.; Jeong, J.; Han, B. S. et al. Subchronic inhalation toxicity of gold nanoparticles. Part. Fibre Toxicol. 2011, 8, 16.

[14] Villiers, C. L.; Freitas, H.; Couderc, R.; Villiers, M. B.; Marche, P. N. Analysis of the toxicity of gold nano particles on the immune system: Effect on dendritic cell functions. $J$. Nanopart. Res. 2010, 12, 55-60.

[15] Sabella, S.; Galeone, A.;Vecchio, G.; Cingolani, R.; Pompa, P. P. AuNPs are toxic in vitro and in vivo: A review. $J$. Nanosci. Lett. 2011, 1, 145-165.

[16] Wang, S.; Lawson, R.; Ray, P. C.; Yu, H. Toxic effects of gold nanoparticles on salmonella typhimurium bacteria. Toxicol. Ind. Health 2011, 27, 0748233710393395.

[17] Zhou, M.; Wang, B. X.; Rozynek, Z.; Xie, Z.; H. Fossum, J. O.; Yu, X. F.; Raaen, S. Minute synthesis of extremely stable gold nanoparticles. Nanotechnology 2009, 20, 505606.

[18] Kanduc, D.; Mittelman, A.; Serpico, R.; Sinigaglia, E.; Sinha, A. A.; Natale, C.; Santacroce, R.; Di Corcia, M. G.; Lucchese, A.; Dini, L. et al. Cell death: Apoptosis versus necrosis (review). Int. J. Oncol. 2002, 21, 165-170.

[19] Pompa, P. P.; Vecchio, G.; Galeone, A.; Brunetti, V.; Maiorano, G.; Sabella, S.; Cingolani, R. Physical assessment of toxicology at nanoscale: Nano dose-metrics and toxicity factor. Nanoscale 2011, 3, 2889-2897. 
[20] Lewinski, N.; Colvin, V.; Drezek, R. Cytotoxicity of nanoparticles. Small 2008, 4, 26-49.

[21] Yah, C. S. The toxicity of gold nanoparticles in relation to their physicochemical properties. Biomed. Res. 2013, 24, 400-413.

[22] Chen, H. J.; Shao, L.; Li, Q.; Wang, J. F. Gold nanorods and their plasmon properties. Chem. Soc. Rev. 2013, 42, 2679 2724.

[23] Aillon, K. L.; Xie, Y.; El-Gendy, N.; Berkland, C. J.; Forrest, M. L. Effects of nanomaterial physicochemical properties on in vivo toxicity. Adv. Drug Delivery Rev. 2009, 61, 457-466.

[24] Khlebtsov, N; Dykman, L. Biodistribution and toxicity of engineered gold nanoparticles: A review of in vitro and in vivo studies. Chem. Soc. Rev. 2011, 40, 1647-1671.

[25] Lewinski, N.; Colvin, V.; Drezek, R. Cytotoxicity of nanoparticles. Small 2008, 4, 26-49.

[26] Pelley, J. L.; Daar, A. S.; Saner, M. A. State of academic knowledge on toxicity and biological fate of quantum dots. Toxicol. Sci. 2009, 112, kfp188.

[27] Maurer-Jones, M. A.; Bantz, K. C.; Love, S. A.; Marquis, B. J.; Haynes, C. L. Toxicity of therapeutic nanoparticles. Nanomedicine 2009, 4, 219-241.

[28] Hussain, S. M.; Braydich-Stolle, L. K.; Schrand, A. M.; Murdock, K. O.; Yu, R. C.; Mattie, D. M.; Schlager, J. J.; Terrones, M. Toxicity evaluation for safe use of nanomaterials: Recent achievements and technical challenges. Adv. Mater. 2009, 21, 1549-1559.

[29] Fadeel B.; Garcia-Bennett, A. E. Better safe than sorry: Understanding properties of inorganic nanoparticles manufactured for biomedical applications. Adv. Drug Delivery Rev. 2010, 62, 362-374.

[30] Johnston, H. J.; Hutchison, G.; Christensen, F. M.; Peters, S.; Hankin, S.; Stone, V. A review of the in vivo and in vitro toxicity of silver and gold nanoparticles: Particle attributes and biological mechanisms responsible for the observed toxicity. Crit. Rev. Toxicol. 2010, 40, 328-346.

[31] Soenen, S. J.; Rivera-Gil, P.; Montenegro, J. M.; Parak, W. J.; De Smedt, S. C.; Braeckmans, K. Cellular toxicity of inorganic nanoparticles: Common aspects and guidelines for improved nanotoxicity evaluation. Nano Today 2011, 6, 446-465.

[32] Rausch, K.; Reuter, A.; Fischer, K.; Schmidt, M. Evaluation of nanoparticle aggregation in human blood serum. Biomacromolecules 2010, 11, 2836-2839.

[33] Cui, W. J.; Li, J. R.; Zhang, Y. K.; Rong, H. L.; Lu, W. S.; Jiang, L. Effects of aggregation and the surface properties of gold nanoparticles on cytotoxicity and cell growth. Nanomedicine: NBM 2012, 8, 46-53.

[34] Pan, Y.; Neuss, S.; Leifert, A.; Wen, F.; Simon, U.; Schmid, G.; Brandau, W.; Jahnen-Dechent, W. Size-dependent cytotoxicity of gold nanoparticles. Small 2007, 3, 1941-1949.

[35] Fratoddi, I.; Venditti, I.; Cametti, C.; Palocci, C.; Chronopoulou, L.; Marino, M.; Acconcia, F.; Russo, M. V. Functional polymeric nanoparticles for dexamethasone loading and release. Colloids Surf., B 2012, 93, 59-66.

[36] Shukla, S.; Priscilla, A.; Banerjee, M.; Bhonde, R. R.; Ghatak, J.; Satyam, P. V. Porous gold nanospheres by controlled transmetalation reaction: A novel material for application in cell imaging. Chem. Mater. 2005, 17, 50005005.

[37] Railsback, J. G.; Singh, A.; Pearce, R. C.; McNight, T. E.; Collazo, R.; Sitar, Z.; Yingling, Y. G.; Melechko, A. V. Weakly charged cationic nanoparticles induce DNA bending and strand separation. Adv. Mater. 2012, 24, 4261-4265.

[38] Paillusson, F.; Dahirel, V.; Jardat, M.; Victor, J. M.; Barbo, M. Effective interaction between charged nanoparticles and DNA. Phys. Chem. Chem. Phys. 2011, 13, 12603-12613.

[39] Poulos, A. S.; Constantin, D.; Davidson, P.;Impéror-Clerc, M.; Pansu, B.; Rouzière, S. The interaction of charged nanoparticles at interfaces. EPL 2012, 100, 18002.

[40] Huang, X. L.; Zhang, B.; Ren, L.; Ye, S. F.; Sun, L. P.; Zhang, Q. Q.; Tan, N. C.; Chow, G. M. In vivo toxic studies and biodistribution of near infrared sensitive Au-AuS nanoparticles as potential drug delivery carriers. J. Mat. Sci.: Mater. Med. 2008, 19, 2581-2588.

[41] Hainfeld, J. F.; Slatkin, D. N.; Focella, T. M.; Smilowitz, H. M. Gold nanoparticles: A new X-ray contrast agent. Br. J. Radiol. 2006. 79, 248-253,

[42] Gerber, A.; Bundschud, M.; Klingelhofer, D.; Groneberg, D. A. Gold nanoparticles: Recent aspects for human toxicology. J. Occup. Med. Toxicol. 2013, 8, 32.

[43] Dreaden, E. C.; Alkilany, A. M.; Huang, X.; Murphy, C. J.; El-Sayed, M. A. The golden age: Gold nanoparticles for biomedicine. Chem. Soc. Rev. 2012, 41, 2740-2779.

[44] Fratoddi, I.; Venditti, I.; Cametti, C.; Russo, M. V. Gold nanoparticles and gold nanoparticle-conjugates for delivery of therapeutic molecules. Progress and challenges. J. Mater. Chem. B 2014, 2, 4204-4220.

[45] Fratoddi, I.; Panziera, N.; Pertici, P.; Martra, G.; Bertinetti, L.; Russo, M. V. Nanostructured gold/conjugated polymer hybrids: Preparation, chemical structure and morphology. Mater. Sci. Engin., C 2007, 27, 1305-1308.

[46] Vitale, F.; Piscopiello, E.; Pellegrini, G.; Fratoddi, I.; Russo, M. V.; Tapfer, L.; Mazzoldi, P. Gold nanoclustersorganometallic polymer nanocomposite: Synthesis and characterization. Mater. Sci. Engin,. C 2007, 27, 1300-1304.

[47] Vitale, F.; Vitaliano, R.; Battocchio, C.; Fratoddi, I.; Piscopiello, E.; Tapfer, L.; Russo, M. V. Synthesis and characterization of gold nanoparticles stabilized by palladium (II) phosphine thiol. J. Organomet. Chem. 2008, 693, 1043- 1048. 
[48] Vitale, F.; Vitaliano, R.; Battocchio, C.; Fratoddi, I.; Giannini, C.; Piscopiello, E.; Guagliardi, A.; Cervellino, A.; Polzonetti, G.; Russo, M. V. et al. Synthesis and microstructural investigations of organometallic Pd(II) thiol-gold nanoparticles. Nanoscale Res. Lett. 2008, 3, 461-467.

[49] Fratoddi, I.; Venditti, I.; Russo, M. V. Breackthroughs for gold nanoparticles:, volume chap. 13. Nova Science Publisher, Inc. NY, 2010. ISBN: 978-1-61668-009-1.

[50] Fratoddi, I.; Venditti, I.; Battocchio, C.; Polzonetti, G.; Cametti, C.; Russo, M. V. Core-shell hybrids based on noble metal nanoparticles and conjugated polymers: Synthesis and characterization. Nanoscale Res. Lett. 2011, 6, 98.

[51] Cametti, C.; Fratoddi, I.; Venditti, I.; Russo, M. V. Dielectric relaxations of thiol-coated noble metal nanoparticles in aqueous solutions. electrical characterization of the interface. Langmuir, 2011, 27, 7084-7090.

[52] Fratoddi, I.; Venditti, I.; Battocchio, C.; Polzonetti, G.; Bondino, F.; Malvestuto, M.; Piscopiello, E.; Tapfer, L.; Russo, M. V. Gold nanoparticle dyads stabilized with binuclear Pt(II) dithiol bridges. J. Phys. Chem. C 2011, 115, 1519815204.

[53] Fratoddi, I.; Battocchio, C.; Polzonetti, G.; Sciubba, F.; Delfini, M.; Russo, M. V. A porphyrin bridged Pd dimer complex stabilizes gold nanoparticles. Eur. J. Inorg. Chem. 2011, 4906-4913.

[54] Quintiliani, M.; Bassetti, M.; Pasquini, C.; Battocchio, C.; Possi, M.; Mura, F.; Matassa, R.; Fontana, L.; Russo, M. V.; Fratoddi, I. Network assembly of gold nanoparticles linked through fluorenyl dithiol bridge. J. Mater. Chem. C 2014, 2, 2517-2527.

[55] Venditti, I.; Fontana, L.; Fratoddi, I.; Battocchio, C.; Cametti, C.; Sennato, S.; Mura, F.; Sciubba, F.; Delfini, M.; Russo, M. V. Direct interaction of hydrophilic gold nanoparticles with dexamethasone drug: Loading and release study. $J$. Colloid Interf. Sci. 2014, 418, 52-60.

[56] Battocchio, C.;Fratoddi, I.; Venditti, I.; Yarzhemsky, V. G.; Norov, Y. V.; Russo, M. V.; Polzonetti, G. Exafs in total refelexion (reflexafs) for the study of organometallic $\mathrm{Pd}(\mathrm{II})$ thiol complexes based self-assembled monolayers on gold. Chem. Phys. 2011, 379, 92-98.

[57] Cedervall, T.; Lynch, I.; Foy, M.; Berggard, T.; Donnelly, S. C.; Cagney, G.; Linse, S.; Dawson, K. A. Detailed identification of plasma proteins adsorbed on copolymer nanoparticles. Angew. Chem. Int. Ed. 2007, 46, 5754-5756.

[58] Lynch, I.; Cedervall, T.; Lundqvist, M.; Cabaleiro-Lago, C.; Linse, S.; Dawson, K. A. The nanoparticle-protein complex as a biological entity; A complex fluids and surface science challenge for the 21st century. Adv. Colloid Interface Sci. 2007, 134-135, 167-174.
[59] Lynch I.; Dawson, K. A. Protein-nanoparticle interactions. Nano Today, 2008, 3, 40-47.

[60] Casals, E.; Pfaller, T.; Duschl, A.; Oosting, G. J.; Puntes, V. Time evolution of the nanoparticle protein corona. ACS Nano. 2010, 4, 3623-3632.

[61] Monopoli, M. P.; Walczyk, D.; Campbell, A.; Elia, G.; Lynch, I.; Bandelli-Bombelli, F.; Dawson, K. A. Physical-chemical aspects of the protein corona: Relevance to in vitro and in vivo biological impacts of nanoparticles. J. Am. Chem. Soc. 2011, 133, 2525-3534.

[62] Perdonet, N.; Fang, X.; Sun, Y.; Bakhtina, A.; Ramakrishnan, A.; Sokolov, J.; Ulman, A.; Rafailovih, M. Adverse effects of citrate-gold nanoparticles on human dermal fibroblasts. Small 2006, 2, 766-773.

[63] Rivera Gil, P.; Huhn, D.; del Mercato, L. L.; Sasse, D.; Parak, W. J. Nanopharmacy: Inorganic nanoscale devices as vectors and active compounds. Pharmacol. Res. 2010, 62, 115125.

[64] Chitharani, B. D.; Ghazani, A. A.; Chan, W. C. W. Determining the size and the shape dependence of gold nanoparticle uptake into mammalian cells. Nano Lett. 2006, 6, 662-668.

[65] Murphy, C. J.; Stone, J. W.; Sisco, P. N.; Alkilany, A. M.; Goldsmith, E. C.; Baxter, S. C. Gold nanoparticles in biology: Beyond toxicity to cellular imaging. Acc. Chem. Res. 2008, 41, 1721-1730.

[66] De Jong, W. H.; Hagens, W. I.; Krystek, P.; Burger, M. C.; Sips, A. J. A. M.; Geertsma, R. E. Particle size-dependent organ distribution of gold nanoparticlesafter intravenous injection. Biomaterials 2008, 29, 1912-1919.

[67] Sonavane, G.; Tomoda, K.; Makino, K. Biodistribution of colloidal gold nanoparticles after intravenous administration: Effect of particle size. Colloids Surf., B 2008, 66, 274-280.

[68] Abdel Halim, M. A. The influence of size and exposure duration of gold nanoparticles on gold nanoparticle levels in several rat organs in vivo. J. Cell Sci. Ther. 2012, 3, 1000129.

[69] Pompa, P. P.; Vecchio, G.; Galeone, A.; Brunetti, V.; Sabella, S.; Maiorano, G.; Falqui, A.; Bertoni, G.; Congolani, R. In vivo toxicity assessment of gold nanoparticles in Drosophila melanogaster. Nano Res. 2011, 4, 405-413.

[70] Simpson, C. A.; Salleng, K. J.; Cliffel, D. E.; Feldheim, D. L. In vivo toxicity, biodistribution, and clearance of glutathione-coated gold nanoparticles. Nanomedicine: NBM 2013, 9, 257-263.

[71] Simpson, C. A.; Huffman, B. J.; Gerdon, A. E.; Cliffel, D. E. Unexpected toxicity of mono layer protected gold clusters eliminated by PEG-thiol place exchange reactions. Chem. Res. Toxicol. 2010, 23, 1608-1616. 
[72] Cazacu, A.; Bindar, D.; Tartau, L.; Hritcu, L.; Stefan, M.; Nita, L.; Ionescu, C.; Nica, V.; Rusu, G.; Dobromir, M. et al. Effect on nerve structures of functionalized gold-chitosan nanoparticles obtained by one pot synthesis. An. Stiint. Univ. “Alexandru Ioan Cuza” Iasi, Sect. II a: Genet. Biol. Mol. 2011, 12, 45-49.

[73] Esumi, K.; Takei, N.; Yoshimura, T. Antioxidant potentiality of gold-chitosan nanocomposites. Colloids Surf. B 2003, 32, 117-123.

[74] Stefan, M.; Melnig, V.; Pricop, D.; Neagu, A.; Mihasan, M.; Tartu, L. Attenuated effects of chitosan-capped gold nanoparticles on LPS-induced toxicity in laboratory rats. Mater. Sci. Eng. C 2013, 33, 550-556.

[75] Coradeghini, R.; Gioria, S.; Garcia, C. P.; Nativo, P.; Franchini, F.; Gilliland, D.; Ponti, J.; Rossi, F. Size-dependent toxicity and cell interaction mechanisms of gold nanoparticles on mouse fibroblasts. Toxicology Lett. 2013, 217, 205-216.

[76] Yamada K. M.; Cukierman, E. Modeling tissue morphogenesis and cancer in 3D. Cell 2007, 130, 601-610.

[77] Lee, J.; Lilly, G. D.; Doty, R. C.; Podsiadlo, P.; Kotov, N. A. In vitro toxicity testing of nanoparticles in $3 \mathrm{D}$ cell culture. Small 2009, 5, 1213-1221.

[78] Ponti, J.; Colognato, R.; Rauscher, H.; Gloria, S.; Broggi, F.; Franchini, F.; Pascual, C.; Guidetti, G.; Rossi, F. Colony forming efficiency and microscopy analysis of multi-well carbon nanotubes cell interactions. Toxicology Lett. 2010, 197, 29-37.

[79] Chen, Y. S.; Hung, Y. C.; Iau, I.; Huang, G. S. Assessement of the in vivo toxicity of gold nanoparticles. Nanoscale Res. Lett. 2009, 4, 858-864.

[80] Sun, L. L.; Liu, D. J.; Wang, Z. X. Functional gold nanoparticle-peptide complexes as cell targeting agents. Langmuir 2008, 24, 10293-10297.

[81] Tkachenko, A. G.; Xie, H.; Liu, Y.; Coleman, D.; Ryan, J.; Glomn, W. R.; Shipton, M. K.; Franzen, S.; Feldheim, D. L. Cellular traiectories of peptide modified gold particle complexes: Comparison of nuclear localization signals and peptide tranduction domain. Bioconjugate Chem. 2004, 15, 482-490.

[82] Khan, J. A.; Pillai, B.; Das, T. K.; Singh, Y.; Maiti, S. Molecular effects of uptake of gold nanoparticles in hela cells. Chem. Biochem. 2007, 8, 1237-1240.

[83] Kuo, C. W.; Lai, J. J.; Wei, K. H.; Chen, P. Studies of surface modified gold nanowires inside living cells. Adv. Funct. Mater. 2007, 17, 3707-3714.

[84] Niidome, T.; Yamagata,M.; Okamoto, Y.; Akiyama, Y.; Takahashi, H.; Kawano, T.; Katayama, Y.; Niidome, Y. PEGmodified gold nanorods with a stealth character for in vivo applications. J. Controlled Rel. 2006, 114, 343-347.
[85] Takahashi, H.; Niidome, Y.; Niidome, T.; Kaneko, K.; Kawasaki, H.; Yamada, S. Modification of gold nanorods using phosphatidylcholine to reduce cytotoxicity. Langmuir 2006, 22, 2-5.

[86] Hauck, T. S.; Ghazani, A. A.; Chan, W. C. W. Assessing the effect of surface chemistry on gold nanorod uptake, toxicity, and gene expression in mammalian cells. Small 2008, 4, 153-159.

[87] Salmaso, S.; Caliceti, P.; Amendola, V.; Meneghetti, M.; Magnusson, J. P.; Pasparakis, G.; Alexander, C. Cell up-take control of gold nanoparticles functionalized with thermoresponsive polymers. J. Mater. Chem. 2009, 19, 1608-1615.

[88] Qu Y. H.; Lü, X. Y. Aqueous synthesis of gold nanoparticles and their cytotoxicity in human dermal fbroblasts fetal. Biomed. Mater. 2009, 4, 025007.

[89] Patra, H. K.; Dasgupta, A. K. Cancer cell response to nanoparticles: Criticality and optimality. Nanomed. Nanotech. Biol. Med. 2012, 8, 842-852

[90] Pérez-Juste, J.; Pastoriza-Santos, I.; Liz-Marzán, L. M.; Mulvaney, P. Gold nanorods: Synthesis, characterization and applications. Coordination Chem. Rev. 2005, 249, 1870-1901.

[91] Alkilany, A. M.; Nagaria, P. K.; Hexel, C. R.; Shaw, T. J.; Murphy, C. J.; Wyatt, M. D. Cellular uptake and cytotoxicity of gold nanorods: Molecular origin of cytotoxicity and surface effects. Small 2009, 5, 701-708.

[92] Huff, T. B.; Hansen, M. N.; Zhao, Y.; Cheng, J. X.; Wei, A. Controlling the cellular uptake of gold nanorods. Langmuir 2007, 23, 1596-1599.

[93] Alkilany, A. M.; Shatanawi, A.; Kurtz, T.; Caldwell, R. B.; Caldwell, R. W. Toxicity and cellular uptake of gold nanorods in vascular endothelium and smooth muscles of isolated rat blood vessel: Importance of surface modification. Small 2012, 8, 1270-1278.

[94] Cortesi, R.; Esposito, E.; Menegatti, E.; Gambari, R.; Nastruzzi, C. Effect of cationic liposome composition on in vitro cytotoxicity and protective effect on carried dna. Int. J. Pharm. 1996, 139, 69-78.

[95] Mirska, D.; Schirmer, K.; Funari, S.; Langner, A.; Dobner, B.; Brezesinski, B. Biophysical and biochemical properties of a binary lipid mixture for dna transfection. Colloids Surf. B 2005, 40, 51-59.

[96] Hirsch, L. R.; Stafford, R. J.; Bankson, J. A.; Sershen, S. R.; Rivera, B.; Price, R. E.; Hazle, J. D.; Halas, N. J.; West, J. L. Nanoshell-mediated near-infrared thermal therapy of tumors under magnetic resonance guidance. Prot. Natl. Acad. Sci. U.S.A. 2003, 100, 13549-13554.

[97] Loo, C.; Lowery, A.; Halas, N.; West, J.; Drezek, R. Immunotargeted nanoshells for integrated cancer imaging and therapy. Nano Lett. 2005, 5, 709-711. 
[98] James, W. D.; Hirsch, L. R.; West, J. L.; O’Neal, P. D.; Payne, J. D. Application of inaa to the build-up and clearance of gold nanoshells in clinical studies in mice. J. Radioanal. Nucl. Chem. 2007, 271, 455-459.

[99] Khlebtsov, N. G.; Dykman, L. A.; Terentyuk, G. S. Iii euroasian congress on medical physics and engineering. In Moscow State Univ. Publ., editor, Med.l Phys.s 2010, 3, 209-211.

[100] Melancon, M. P.; Lu, W.; Yang, Z.; Zhang, R.; Cheng, Z.; Elliot, A. M.; Stafford, J.; Olson, T.; Zhang, J. Z.; Li, C. In vitro and in vivo targeting of hollow gold nanoshells directed at epidermal growth factor receptor for photothermal ablation therapy. Mol. Cancer Ther. 2008, 7, 1730-1739.

[101] Su, C. H.; Sheu, H. S.; Lin, C. Y.; Huang, C. C.; Lo, Y. W.; Pu, Y. C.; Weng, J. C.; Shieh, D. B.; Chen, J. H.; Yeh, C. S. Nanoshell magnetic resonance imaging contrast agents. J. Am. Chem. Soc. 2007, 129, 2139-2146.

[102] Xia, Y. N; Li, W. Y.; Cobley, C. M.; Chen, J. Y.; Xia, X. H.; Zhang, Q.; Yang, M. X.; Cho, E. C.; Brown, P. K. Gold nanocages: From synthesis to theranostic applications. Acc. Chem. Res. 2011, 44, 914-924.

[103] Dykman, L. A.; Khlebtson, N. G. Gold nanoparticles in biomedical applications: Recent advances and perspectives. Chem. Soc. Rev. 2012, 41, 2256-2282.

[104] Yang, X. M.; Stein, E. W.; Ashkenazi, S.; Wang, L. V. Nanoparticles for photoacoustic imaging. Wiley Interdiscip. Rev.: Nanomed. Nanobiotechnol. 2009, 1, 360-368.

[105] Wang, Y. C.; Liu, Y. J.; Luehmann, H.; Xia, X. H.; Ean, D. H.; Cutler, C.; Xia, Y. N. Radioluminescent gold nanocages with controlled radioactivity for real-time in vivo imaging. Nano Lett. 2013, 13, 581-585.

[106] Kim, C.; Cho, E. C.; Chen, J. Y.; Song, K. H.; Au, L.; Favazza, C.; Zhang, Q.; Cobley, C. M.; Gao, F.; Xia, Y. $\mathrm{N}$. et al. In vivo molecular photoacustic tomography of melanomas targeted by bioconjugated gold nanogages. ACS Nano 2010, 4, 4559-4564.

[107] Chen, J. Y.; Glaus, C.; Laforest, R.; Zhang, Q.; Yang, M. X.; Gidding, M.; Welch, M. J.; Xia, Y. N. Gold nanocages as photothermal transducer for cancer treatment. Small 2010, 6, 811-817.

[108] Au, L.; Zhang, Q.; Cobley, C. M.; Gidding, M.; Schwartz, A. G.; Chen, J. Y.; Xia, Y. N. Quantifying the cellular uptake of antibody-conjugated Au nanocages by twophoton microscopy and inductively coupled plasma mass spectrometry. ACS Nano 2010, 4, 35-42.

[109] Rodriguez-Lorenzo, L.; Alvarez-Puebla, R. A.; Garcia de Abajo, F. J.; Liz-Marzan, L. M. Surface enhanced raman scattering using star-shaped gold colloidal nanoparticles. J. Phys. Chem. C 2010, 114, 7336-7340.
[110] Yuan, H.; Khoury, C. G.; Hwang, H.; Wilson, C. M.; Grant, G. A.; Vo-Dinh, T. Gold nanostars: Surfaxctant free synthesis, 3D modelling and two photon photoluminescence imaging. Nanotechnology 2012, 23, 075102.

[111] Trigari, S.; Rindi, A.; Margheri, G.; Sottini, S.; Dellapiane, G.; Giorgetti, E. Synthesis and modelling of gold nanostars with tunable morphology and extinction spectrum. J. Mater. Chem. 2011, 21, 6531-6549.

[112] Salinas, K.; Kereselidze, Z.; De Luna, F.; Peralta, X. G.; Santamaria, F. Transient extracellular application of nanostars increase hippocampal neuronal activity. J. Nanobiotechnology 2014, 12, 31-38.

[113] Navarro, J. R. G.; Manchon, D.; Lerouge, F.; Blanchard, N. P.; Marotte, S.; Leverrier, J.; Marvel, J.; Chaput, F.; Micouin, G.; Gabudean, A. A. et al. Synthesis of pegylated gold nanostares and bipyramids for intracellular uptake. Nanotechnology 2012, 23, 465602.

[114] Dam, D. H. M.; Lee, R. C.; Odom, T. W. Improved in vitro efficacy of gold nanoconstructs by increased loading of g-quadruplex aptamer. Nano Lett. 2014, 14, 2843-2848.

[115] Dam, D. H. M.; Culver, K. S. B.; Odom, T. W. Grafting aptamers onto gold nanostars increases in vitro efficacy in a wide range of cancer cell types. Mol. Pharmaceutics 2014, 11, 580-587.

[116] Hutter, E.; Boridy, S.; Labrecque, S.; Lalancette-Hebert, M.; Kriz, J.; Winnik, F. M.; Maysinger, D. Microglial response to gold nanoparticles. ACS Nano 2010, 4, 2595-2606.

[117] Li, W. T.; Sun, X. L.; Wang, Y.; Niu, G.; Chen, X. Y.; Qian, Z. Y.; Nie, L. M. In vivo quantitative photoacustic microscopy of gold nanostar kinetics in mouse organ. Biomed. Optics Express 2014, 5, 2679-2685.

[118] Rivera-Gil, P.; Jamenez-de Aberasturi, D.; Wulf, V.; Pelaz, B.; Del Pino, P.; Zhao, Y.; De La Fluente, J. M.; Ruiz de Larramendi, I.; Liang, X. J.; Parak, W. J. The challenge to relate the physicochemical properties of colloidal nanoparticles to their cytotoxicity. Acc. Chem. Res. 2013, 46, 743-749.

[119] Johnston, H.; Pojana, G.; Zuin, S.; Jacobsen, N. R.; Moller, P.; Loft, S.; Semmler-Behnke, M.; McGuiness, C.; Balharry, D.; Marcomini, A. et al. Engineered nanomaterial risk. essons from completed nanotoxicology studies: Potential solutions to current and future challenges. Crit. Rev. Toxicol. 2013, 43, 1-20.

[120] Roebben, G.; Ramirez-Garcia, S.; Hackley, V. A.; Roesslein, M.; Klaessig, F.; Kestens, V.; Lynch, I.; Garner, C. M.; Rawle, A.; Elder, A. et al. Interlaboratory comparison of size and surface charge measurements on nanoparticles prior to biological impact assessment. J. Nanopart. Res. 2011, 13, 2675-2687. 
[121] Oberdorster, G.; Oberdorster, E.; Oberdorster, J. Nanotoxicity: An emerging discipline evolving from studies of ultrafine particles. Environ. Health Perspect. 2005, 113, 823-839.

[122] Tedesco, S.; Doyle, H.; Redmond, G.; Sheehan, D. Gold nanoparticles and oxidative stress in mytilus edulis. Marine Environ. Res. 2008, 66, 131-133.

[123] Kong, B.; Seog. J. H.; Graham, L. M.; Lee, S. B. Experimental considerations on the cytotoxicity of nanoparticles. Nanomedicine, 2011, 6, 929-941.

[124] Malugin, A.; Ghandehari, H. Cellular uptake and toxicity of gold nanoparticles in prostate cancer cells: A comparative study of rods and spheres. J. Appl. Toxicol. 2010, 30, 212-217.

[125] Albanese A.; Chan, W. C. Effect of gold nanoparticles aggregation on cell uptake and toxicity. ACS Nano 2011, 5, 5478-5489.

[126] Elsaesser, A.; Howard, C. V. Toxicology of nanoparticles. Adv. Drug Deliv. Rev. 2012, 64, 129-137.

[127] Oberdörster, G. Safety assessment for nanotechnology and nanomedicine: Concepts of nanotoxicology. J. Intern. Med. 2010, 267, 89-105.

[128] Oberdöster, G.; Mayriard, A.; Donaldson, K.; Castranova, V.; Fitzpatrick, J.; Ausman, K.; Carter, J.; Karn, B.; Kreyling, W.; Lai, D. et al. Principles for charcterizing the potential human health effects from exposure to nanoparticles: Elements for a screening strategy. Part. Fibre Toxicol. 2005, 2, 8-1/35.

[129] Elsaesser, A.; Taylor, A.; de Yanés, G. S.; McKerr, G.; Kim, E. M.; O’Hare, E.; Howard, C. V. Quantification of nanoparticle uptake by cells using microscopical and analytical techniques. Nanomedicine 2010, 5, 1447-1457.

[130] Wittmaack, K. In search of the most relevant parameters for quantifying lung inflannatory response to nanoparticle exposure: Particle number, surface area or what ? Environ. Health Perspect. 2007, 115, 187-194.

[131] Joris, F.; Manshian, B. B.; Peynshaert, K.; De Smedt, S. C.; Braeckman, K.; Soenen, S. J. Assessing nanoparticle toxicity in cell-dased assays: Influence of the cell culture parameters and optimized models for bridging the in vitroin vivo gap. Chem. Soc. Rev. 2013, 42, 8339-8359.

[132] Rushton, E. K.; Jiang, J.; Leonard, S. S.; Eberly, S.; Castranova, V.; Biswas, P.; Elder, A.; Han, X.; Gelein, R.; Finkelstein, J.; Oberdorster, G. Concept of assessing nanoparticle hazards considering nanoparticle dosemetric and chemical/biological response metrics. J. Toxicol. Environ. Health, Part A 2010, 73, 445-461.

[133] Gulson. B.; Wong, H. Stable isotopic tracing: A way forward for nanotoxicology. Environ. Health Perspect. 2006, 114, 1486-1488.
[134] Wang, L. M.; Li, Y. F.; Zhou, L. J.; Liu, Y.; Meng, L.; Zhang, K.; Wu, X. C.; Zhang, L. L.; Li, B.; Chen, C. Y. Characterization of gold nanorods in vivo by integrated analytical techniques: Their uptake, retention and chemical forms. Anal. Bioanal. Chem. 2010, 396, 1105-1114.

[135] Darien, B. J.; Sims, P. A.; Kruse-Elliott, K. T.; Homan, T. S.; Cashwell, R. J.; Albrecht, R. M. Use of colloidal gold and neutron activation in correlative microscopic labeling and label quantitation. Scanning Microsc. 1995, 9, 773780.

[136] Qiu, Y.; Liu, Y.; Wang, L. M.; Xu, L. G.; Bai, R.; Ji, Y. L.; Wu, X. C.; Zhao, Y. L.; Li, Y. F.; Chen, C. Y. Surface chemistry and aspect ratio mediated cellular uptake of $\mathrm{Au}$ nanorods. Biomaterials 2010, 31, 7606-7619.

[137] Lehmann, A. D.; Parak, W. J.; Zhang, F.; Ali, Z.; Röcker, C.; Nienhaus, G. U.; Gehr, P.; Rothen-Rutishauser, B. Fluorescent magnetic nanoparticles induce a dose dependent increase in proinflammatory response in lung cells in vitro correlated with intracellular localization. Small 2010, 6, 753-762.

[138] Teeguarden, J. G.; Hinderliter, P. M.; Orr, G.; Thrall, B. D.; Pounds, J. G. Particokinetics in vitro: Dosimetry considerations for in vitro nanoparticle toxicity assessments. Toxicol. Sci. 2007, 95, 300-312.

[139] Alvarez, P. J. J.; Colvin, V.; Lead, J.; Stone, V. Research priorities to advance eco-responsible nanotechnology. ACS Nano 2009, 3, 1616-1619.

[140] Chithrani, B. D.; Chang, W. C. W. Elucidating the mechanism of cellular uptakeand removal of protein-coated gold nanoparticles of different sizes and shapes. Nano Lett. 2007, 7, 1542-1550.

[141] Green, J. J.; Chiu, E.; Leshchiner, E. S.; Shi, J.; Langer, R.; Anderson, D. G. Electrostatic ligand coatings of nanoparticles enable ligand-specific gene delivery to human primary cells. Nano Lett. 2007, 7, 874-879.

[142] Marquis, B. J.; Love, S. A.; Brown, K. L.; Haynes, C. L. Analytical methods to assess nanoparticle toxicity. Analyst 2009, 134, 425-439.

[143] Soenen, S. J.; De Cuyper, M. Assessing cytotoxicity of (iron-oxide based) nanoparticles: An overwiew of different methods exemplified with cationic magnetoliposomes. Contrast Media Mol. Imaging 2009, 4, 207-219.

[144] Monteiro-Riviere, N. A.; Inman, A. O.; Zhang, L. W. Limitations and relative utility of screening assay to assess engineered nanoparticle toxicity in a human cell line. Toxicol. Appl. Pharmcol. 2009, 234, 222-235.

[145] Dhawan, A.; Sharma, V. Toxicology assessment of nanoparticles: Methods and challenges. Anal. Bioanal. Chem. 2010, 398, 589-605. 
[146] Nel, A. E.; Madler, L.; Velegol, D.; Xia, T.; Hoek, E. M.; Somasundaran, P.; Klaessig, F.; Castranova, V.; Thompson, M. Understanding biophysicochemical interactions at the nano-bio interfaces. Nat. Mater. 2009, 8, 543-557.

[147] Torrano, A. A.; Pereira, A. S.; Oliviera, O. N.; BarrosTimmons, A. Probing the interactions of oppositely charged gold nanoparticles with dppg and dppc langmuir monolayers as cell membrane models. Colloids Surf., B 2013, 108, 120-126.

[148] Negoda, A.; Liu, Y.; Hou, W. C.; Corredor, C.; Moghadam, B. Y.; Mussolf, C.; Li, W.;Walker, L.; Westerhoff, P.; Mason, A. J.et al. Engineered nanomaterials interactions with bilayer lipid membranes: Screening platforms to assess nanoparticle toxicity. Int. J. Biomed. Nanosci. Nanothechnol. 2013, 3, 52-83.

[149] Chen, K. L.; Bothun, G. D. Nanoparticles meet cell membranes: Probing non specific interactions using model membranes. Environ. Sci. Technol. 2014, 48, 873880.

[150] Podila, R.; Brown, J. M. Toxicity of engineered nanomaterials: A physicochemical perspective. J. Biochem. Mol. Toxicol. 2013, 27, 50-55.

[151] Xu, M. S.; Fujita, D.; Kajiwara, S.; Minowa, T.; Li, X. L.; Takemura, T.; Iwai, H.; Hanagata, N. Contribution of physicochemical characterization of monooxides to cytotoxicity. Biomaterials 2010, 31, 8022-8031.

[152] Fenoglio, I.; Greco, G.; Tomatis, M.; Muller, J.; RaymondoPinero, E.; Béguin, F.; Fonseca, A.; Nagy, J. B.; Lison, D.; Fubini, B. Structural defects play a major role in the acute lung toxicity of multiwalled carbon nanotubes: physicochemical aspects. Chem. Res. Toxicol. 2008, 21, 1690-1697.

[153] National Research Council. Toxicity testing in the 21st century: A vision and strategy, National Academies Press, Washington, DCEdition, 2007. available at http://dels.nas.edu/.

[154] National Research Council. Toxicity in the 21st century: The role of the national toxicology program, National Academies Press, Washington, DCEdition, 2004. available at http://ntp.niehs.nih.gov/.

[155] Sun, B. B.; Li, R. B.; Wang, X.; Xia, T. Predictive toxicological paradigm and high throughput approach for toxicity screening of engineered nanomaterials. Int. $J$. Biomed. Nanosci. Nanothechnol. 2013, 3, 4-18.

[156] Kim, E. Y.; Schulz, R.; Swantek, P.; Kunstman, K.; Malim, M. H.; Wolinsky, S. M. Gold nanoparticle-mediated gene delivery induces widespread changes in the expression of innate immunity genes. Gene Ther. 2012, 19, 347-353.

[157] Patel, S.; Jung, D.; Yin, P. T.; Carlton, P.; Yamamoto, M.; Bando, T.; Sugiyama, H.; Lee, K. B. Nanoscript: A nanoparticle-based artificial transcription factor for effective gene delivey. ACS Nano 2014, 8, 8959-8967.

[158] Rauch, J.; Kolch. W.; Laurent, S.; Mahmoudi, M. Big signals from small particles: Regulation of cell signaling pathways by nanoparticles. Chem. Rev. 2013, 113, 3391-3406

[159] Xue, J. P.; Shan, L. L.; Chen, H. Y.; Li, Y.; Zhu, H. Y.; Deng, D. W.; Qian, Z. Y.; Achilefu, S.; Gu, Y. Q. Visual detection of STAT5B gene expression in living cells using the hairpin DNA modified gold nanoparticles. Biosens. Bioelectron. 2013, 41, 71-77. 



\section{Table of contents}

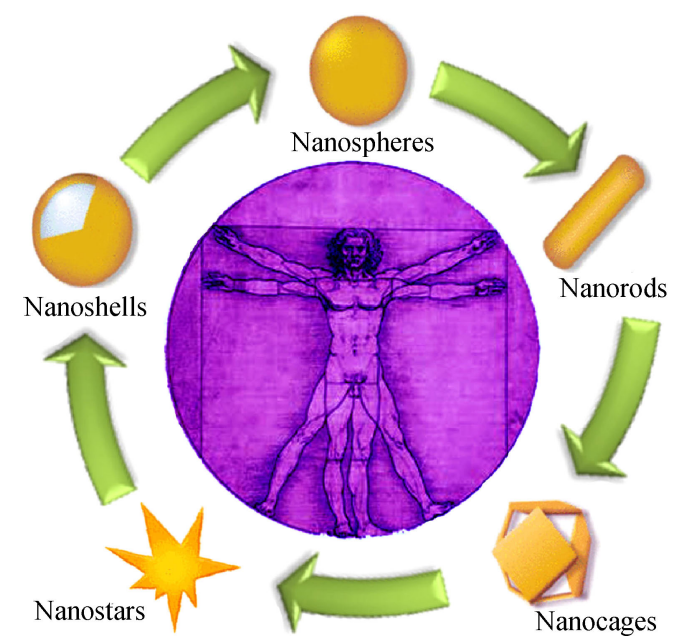

Toxic or beneficial effects of gold nanoparticles on human health depend on their shape, surface charge, and functionalization and biological viability. Widely accepted laboratory research protocols are recommended to overcome the spread of controversial results. 